\title{
Low frequency follow up of radio haloes and relics in the GMRT Radio Halo Cluster Survey
}

\author{
T. Venturi ${ }^{1}$, S. Giacintucci ${ }^{2,3}$, D. Dallacasa ${ }^{4}$, R. Cassano ${ }^{1}$, G. Brunetti ${ }^{1}$, G. Macario $^{5}$, and R. Athreya ${ }^{6}$ \\ 1 INAF - Istituto di Radioastronomia, via Gobetti 101, 40129 Bologna, Italy \\ e-mail: tventuri@ira.inaf.it \\ 2 Department of Astronomy, University of Maryland, College Park, MD 20742-2421, USA \\ 3 Joint Space-Science Institute, University of Maryland, College Park, MD 20742-2421, USA \\ 4 Dipartimento di Astronomia, Università di Bologna, via Ranzani 1, 40126 Bologna, Italy \\ 5 Laboratoire Lagrange, UMR7293, Université de Nice Sophia-Antipolis, CNRS, Observatoire de la Côte d'Azur, 06300 Nice, France \\ ${ }^{6}$ Indian Institute of Science Education and Research (IISER), Pune, India
}

Received 22 June 2012 / Accepted 24 October 2012

\section{ABSTRACT}

\begin{abstract}
Aims. To gain insight into the origin of diffuse radio sources in galaxy clusters and their connection with cluster merger processes, we performed GMRT low frequency observations of the radio haloes, relics and new candidates belonging to the GMRT radio Halo cluster sample first observed at $610 \mathrm{MHz}$. Our main aim was to investigate their observational properties and integrated spectra at frequencies below $610 \mathrm{MHz}$.

Methods. High sensitivity imaging was performed using the GMRT at $325 \mathrm{MHz}$ and $240 \mathrm{MHz}$. The properties of the diffuse emission in each cluster were compared to our $610 \mathrm{MHz}$ images and/or literature information available at other frequencies, in order to derive the integrated spectra over a wide frequency range.

Results. Cluster radio haloes form a composite class in terms of spectral properties. Beyond the classical radio haloes, whose spectral index $\alpha$ is in the range $\sim 1.2 \div 1.3\left(S \propto v^{-\alpha}\right)$, we found sources with $\alpha \sim 1.6 \div 1.9$. This result supports the idea that the spectra of the radiating particles in radio haloes is not universal and that inefficient mechanisms of particle acceleration are responsible for their origin. We also found a variety of brightness distributions, i.e. both centrally peaked and clumpy haloes. Even though the thermal and relativistic plasma tend to occupy the same cluster volume, in some cases a positional shift between the radio and X-ray peaks of emission is evident. Our observations also revealed diffuse cluster sources that cannot be easily classified as either haloes or relics. New candidate relics were found in A 1300 and in A 1682, and in some clusters "bridges" of radio emission have been detected, connecting the relic and radio halo emission. Finally, by combining our new data with information in the literature, we derived the $\log L_{\mathrm{X}}-\log P_{325 \mathrm{MHz}}$ correlation for radio haloes, and investigated the possible correlation of the spectral index of radio haloes with the temperature of the intracluster medium.
\end{abstract}

Key words. radio continuum: galaxies - galaxies: clusters: general

\section{Introduction}

Our understanding of the interplay between the thermal and nonthermal components in clusters of galaxies, as well as their link to the processes leading to the formation of massive clusters in the hierarchical scenario, has considerably improved thanks to the high sensitivity of radio interferometers, such as the Very Large Array (VLA) and the Giant Metrewave Radio Telescope (GMRT), and to the advent of the Chandra and XMM-Newton $\mathrm{X}$-ray observatories.

Non-thermal radio emission in galaxy clusters may take the form of radio haloes and relics, which are diffuse and extended sources (up and above the $\mathrm{Mpc}$ ) of low surface brightness (of the order of the $\mu \mathrm{Jy} \operatorname{arcsec}^{-2}$ ) with no obvious optical counterpart, which have so far been found in about 40 to 50 clusters. They prove the existence of relativistic particles (with Lorentz factor $\gamma \gg 1000$ ) and of large-scale $\mu \mathrm{G}$ magnetic fields. Their powerlaw radio spectrum, with typical spectral index $\alpha \sim 1.2-1.3^{1}$ is the signature of a synchrotron origin (see Ferrari et al. 2008; Venturi 2011; and Feretti et al. 2012 for recent observational reviews). Beyond the similar observational properties, haloes and

$1 S_{v} \propto v^{-\alpha}$, where $S_{v}$ is the flux density at the frequency $v$. relics differ in the location within the cluster and in their polarization properties. Radio haloes are centrally located, with a rather regular shape usually coincident with the distribution of the intracluster medium (ICM) and are unpolarized (exception made for MACS J0717.5+3745, Bonafede et al. 2009), suggesting that the radio emission comes from a region that is co-spatial with the X-ray emitting gas. On the other hand, relics are found in peripheral cluster regions and usually exhibit an elongated structure and high fractional polarization (e.g., Clarke \& Enßlin 2006; van Weeren et al. 2010), which suggests that they are located in external regions of the clusters or seen in projection on the cluster centre.

The longstanding key question concerns the origin of haloes and relics, since the radiative life-time of the radiating electrons is much shorter than the diffusion time needed to cover the cluster scale volumes, therefore some form of in-situ particle acceleration is needed to account for their existence (Jaffe 1977; see also Cassano 2009; and Brunetti 2011, for recent reviews).

All models proposed to explain the origin of relic sources invoke the presence of a shock in the thermal gas, possibly induced by dynamical activity within the cluster (Enßlin et al. 1998; Enßlin \& Gopal-Kishna 2001; Hoeft \& Brüggen 2007). So 
Table 1. Clusters observed with the GMRT.

\begin{tabular}{lcccccc}
\hline \hline Cluster name & $\begin{array}{c}\text { RA } \\
(\mathrm{h} \mathrm{m} \mathrm{s})\end{array}$ & $\begin{array}{c}\text { Dec } \\
\left({ }^{\circ}{ }^{\prime \prime}\right)\end{array}$ & $z$ & Radio source & Ref. & $\begin{array}{c}\text { Scale } \\
\left(\mathrm{kpc} /{ }^{\prime \prime}\right)\end{array}$ \\
\hline A 2744 & 001418.8 & -302300 & 0.307 & GRH & $\mathrm{a}$ & 4.526 \\
A 209 & 013153.0 & -133634 & 0.206 & GRH & $\mathrm{a}$ & 3.377 \\
A 521 & 045409.1 & -101419 & 0.248 & GRH+Rel & b, c, d & 3.887 \\
A 697 & 084253.3 & +362012 & 0.282 & GRH & e & 4.265 \\
A 781 & 092023.2 & +302615 & 0.298 & Rel+Candidate RH & $\mathrm{f}$ & 4.434 \\
Z2661 & 094957.0 & +170858 & 0.383 & Candidate RH & $\mathrm{a}$ & 5.231 \\
A 1300 & 113156.3 & -195537 & 0.308 & RH+Rel & $\mathrm{a}$ & 4.536 \\
A 1682 & 130649.7 & +463259 & 0.226 & Candidate RH+Rel & $\mathrm{a}, \mathrm{g}$ & 3.626 \\
RXCJ 1314.4-2515 & 131428.0 & -251541 & 0.244 & RH+Double Rel & $\mathrm{a}, \mathrm{h}$ & 3.840 \\
A 1758N & 133232.1 & +503037 & 0.280 & RH & $\mathrm{a}$ & 4.244 \\
RXCJ 2003.5-2323 & 200330.4 & -232305 & 0.317 & GRH & $\mathrm{i}$ & 4.625 \\
\hline
\end{tabular}

References. a) This paper; b) Giacintucci et al. (2008); c) Brunetti et al. (2008); d) Dallacasa et al. (2009); e) Macario et al. (2010); f) Venturi et al. (2011a); g) Venturi et al. (2011b); h) Mazzotta et al. (2011); i) Giacintucci et al. (2009).

far a spatial connection between a X-ray shock, or candidate, and cluster-scale radio emission in the form of a distinct radio relic, or an edge in the radio halo structure, has only been reported for a handful of clusters: the Bullet cluster (Markevitch et al. 2002), A 520 (Markevitch et al. 2005); A 754 (Macario et al. 2011a); A 3667 (Finoguenov et al. 2010; Akamatsu et al. 2012); A 521 (Giacintucci et al. 2008; Bourdin et al. 2012); RXCJ 1314.52515 (Mazzotta et al. 2011). A census of the relics studied so far is given in van Weeren et al. (2011a); and a review of our knowledge of shocks and of the related radio emission has been recently given in Markevitch (2010).

The origin of giant radio haloes has been more debated. A popular scenario, the so-called "re-acceleration model" (a modern elaboration of the class of the "in-situ" acceleration scenario, first proposed by Jaffe 1977, and Roland 1981; and quantitatively developed by Schlickeiser et al. 1987), assumes that relativistic electrons are re-accelerated in the ICM by magnetohydrodynamic turbulence injected in the cluster volume by merger events (Brunetti et al. 2001; Petrosian 2001; Fujita et al. 2003). This scenario is motivated by the observed connection between radio haloes and cluster mergers and by considerations of the spectra of radio haloes, which appear to favour mechanisms that are not very efficient in the acceleration of the emitting particles (see Brunetti 2011, for a recent overview). Alternative models assume that the relativistic electrons are secondary products of the collisions between the intergalactic cosmic rays and the thermal protons in the ICM (the so-called "secondary models", see Dennison 1980; Blasi \& Colafrancesco 1999; Pfrommer \& Enßlin 2004; Keshet \& Loeb 2010). These models are based on the argument of cosmic ray confinement in the intracluster medium, and on the fact that the production of secondary electrons, at least to some extent, is unavoidable. More recently, turbulent re-acceleration of relativistic protons and secondary particles has also been investigated (Brunetti \& Blasi 2005; Brunetti \& Lazarian 2011).

The GMRT Radio Halo Survey (Venturi et al. 2007, 2008, V07 and V08, respectively) has provided a first insight into the statistical properties of radio haloes. It has been shown that they are transient phenomena, being hosted only in $\sim 30 \%$ of all massive clusters (Cassano et al. 2006, and 2008; Cassano 2009) that undergo major mergers, as quantitatively derived from the analysis of the X-ray emission. Conversely, to date no haloes have been detected in dynamically relaxed clusters (Cassano et al. 2010). These results support the importance of turbulence in re-accelerating and confining the emitting particles in cluster mergers (Brunetti et al. 2009; Enßlin et al. 2011).

Turbulent re-acceleration models furthermore predict the existence of a composite population of radio haloes, with a spectral index steeper than studied so far at centimetre wavelengths. It is now clear that such sources exist: the radio halo in A 521, with a spectral slope $\alpha \sim 1.9$, is considered the prototype of these "ultra-steep spectrum radio haloes" (USSRH) (Brunetti et al. 2008; Dallacasa et al. 2009). The current status of our knowledge of the diffuse sources in galaxy clusters can be found in recent conference proceedings on this topic (Ferrari et al. 2011; Dwarakanath et al. 2011).

The largest majority of radio haloes and relics have been detected and imaged at $1.4 \mathrm{GHz}$ (i.e. Bacchi et al. 2003; Govoni et al. 2001, and 2004; Giovannini et al. 2009), and interferometric high sensitivity observations at frequencies $v \leq 325 \mathrm{MHz}$ are only available in a handful of cases. As a result, our knowledge of the properties of haloes and relics and of their spectra at frequencies below $v \sim 325 \mathrm{MHz}$ is still poor and incomplete. To start filling this gap, we performed high sensitivity, low frequency follow-up observations of all the cluster sources (radio haloes and relics) and candidate diffuse emission detected with the GMRT at $610 \mathrm{MHz}$, as well as the known radio haloes in the GMRT cluster sample that still lack information at frequencies below $1.4 \mathrm{GHz}$ (V07 and V08). The clusters were surveyed at $325 \mathrm{MHz}$, and some of them were followed up at $240 \mathrm{MHz}$ as well.

In this paper we report on the results of our $325 \mathrm{MHz}$ and $240 \mathrm{MHz}$ GMRT observations. The paper is organised as follows. In Sect. 2 we present the radio observations and data reduction; in Sect. 3 we present the $325 \mathrm{MHz}$ and $240 \mathrm{MHz}$ radio analysis of the radio haloes and diffuse sources; in Sect. 4 we discuss our results. Conclusions are given in Sect. 5 .

We adopt the $\Lambda \mathrm{CDM}$ cosmology, with $H_{0}=$ $70 \mathrm{~km} \mathrm{~s}^{-1} \mathrm{Mpc}^{-1}, \Omega_{\mathrm{m}}=0.3$ and $\Omega_{\Lambda}=0.7$.

\section{Observations and data reduction}

\subsection{The cluster sample}

From the GMRT radio halo cluster sample (V07 and V08) we selected all clusters with a diffuse source, either in the form of a radio halo or relics, and those with "suspect" diffuse emission, for a low frequency follow up. Table 1 provides the list of all 
Table 2. Details of the GMRT observations presented in this paper.

\begin{tabular}{lccccc}
\hline \hline Cluster name & $\begin{array}{c}v \\
(\mathrm{MHz})\end{array}$ & $\begin{array}{c}\Delta v \\
(\mathrm{MHz})\end{array}$ & $\begin{array}{c}\text { Obs. time } \\
(\mathrm{h})\end{array}$ & $\begin{array}{c}\mathrm{HPBW}^{\prime} \mathrm{PA}^{a} \\
\left({ }^{\prime \prime} \times^{\prime \prime},{ }^{\circ}\right)\end{array}$ & $\begin{array}{c}\mathrm{rms} \\
\left(\mathrm{mJy} \mathrm{b}^{-1}\right)\end{array}$ \\
\hline A 2744 & 325 & 32 & 8 & $15.9 \times 8.5,38$ & 0.15 \\
A 209 & 325 & $32^{b}$ & 8 & $11.0 \times 8.7,30$ & 0.12 \\
Z 2661 & 325 & 32 & 10 & $10.8 \times 8.4,-83$ & 0.12 \\
A 1300 & 325 & $32^{b}$ & 8 & $14.0 \times 8.8,8$ & 0.45 \\
A 1682 & 240 & 8 & 6 & $13.4 \times 10.1,47$ & 0.58 \\
RXCJ 1314.4-2515 & 325 & 32 & 8 & $15.1 \times 8.0,32$ & 0.16 \\
A 1758N & 325 & 32 & 8 & $10.5 \times 8.4,58$ & 0.10 \\
\hline
\end{tabular}

Notes. ${ }^{(a)}$ Half-power beamwidth and position angle of the full array; ${ }^{(b)}$ the observations were carried out using a total bandwidth of $32 \mathrm{MHz}$ (USB+LSB), but only the USB was used for the analysis.

clusters in our project. The legend to Col. 5 is: $\mathrm{GRH}=$ giant radio halo ${ }^{2} ; \mathrm{RH}=$ radio halo; $\mathrm{Rel}=$ relic.

Four clusters in Table 1 have been published in dedicated works: A 521 (Giacintucci et al. 2008; Brunetti et al. 2008), A 697 (Macario et al. 2010), A 781 (Venturi et al. 2011a), and RXCJ 2003.5-2323 (Giacintucci et al. 2009). In this paper we present the remaining clusters, and we incorporate all the clusters in Table 1 in the discussion.

\subsection{Data reduction and imaging}

All clusters except A 1682 were observed at $325 \mathrm{MHz}$ for a total of approximately eight hours, using both the upper and lower side bands (USB and LSB, respectively), left and right polarization, for a total observing bandwidth of $32 \mathrm{MHz}$. The data were recorded in spectral-line mode with 128 channels/band, leading to a spectral resolution of $125 \mathrm{kHz} / \mathrm{channel}$. A 1682 was observed for $\sim 6 \mathrm{~h}$ in dual band at $240 / 610 \mathrm{MHz}$ as part of a $610 \mathrm{MHz}$ re-observation $(610 \mathrm{MHz}$ data presented in V08), with a $240 \mathrm{MHz}$ bandwidth of $8 \mathrm{MHz}$ spread over 64 spectral channels. Night observing was carried out for all sources, to minimize the effects of ionosphere and radio frequency interference (RFI). Table 2 reports the observational details.

The USB and LSB datasets were calibrated and reduced individually using the NRAO Astronomical Image Processing System (AIPS) package. Strong RFI affected each observation, and in some cases only one portion of the band was used to produce the final images (see individual comments in the next section and notes to Table 2). For this reason, beyond the standard flagging of bad baselines, antennas, and time ranges, we carried out a very accurate inspection of each dataset in order to identify and remove those data affected by RFI. In all cases the bandpass calibration was performed using the flux density calibrator. The calibration solutions were applied to the data by running the AIPS task FLGIT, which subtracts a continuum from the channels in the $u-v$ plane, determined on the basis of the bandpass shape and using a specified set of channels. Those data whose residuals exceeded a chosen threshold were flagged.

Each dataset was then averaged to six channels of $\sim 2 \mathrm{MHz}$ each. Given the large field of view of the GMRT, each step of the self-calibration cycle was carried out by means of the wide-field imaging technique to account for the non-planar nature of the sky. We used 25 facets covering a total field of view of $\sim 2.7^{\circ} \times$ $2.7^{\circ}$. In those cases where both USB and LSB could be used,

\footnotetext{
2 Linear size $\geq 1 \mathrm{Mpc}$ as defined in CB05, with $H_{0}=50 \mathrm{~km} \mathrm{~s}^{-1} \mathrm{Mpc}^{-1}$. This size corresponds to $\gtrsim 700 \mathrm{kpc}$ with the cosmology assumed in this paper.
}

after a number of phase self-calibration cycles, the final USB and LSB datasets were averaged further from six channels to one single channel, and then combined to produce the final images. Bandwidth smearing is only relevant at the edge of the wide field of view at $325 \mathrm{MHz}$ and $240 \mathrm{MHz}$, and it does not affect the central cluster regions presented and analysed in this paper.

Despite the massive editing required by the RFI in some cases, the quality of the images is generally good, with the $1 \sigma \mathrm{rms}$ level in the range $0.1-0.5 \mathrm{mJy} \mathrm{beam}^{-1}$. The rms values reported in Table 2 were measured on the full resolution images. We estimate that the residual amplitude errors are $\$ 5 \%$.

For all clusters we produced sets of final images, from the full GMRT resolution ( $10^{\prime \prime}$ at $325 \mathrm{MHz}$, and $\sim 13^{\prime \prime}$ at $\left.240 \mathrm{MHz}\right)$ to tapered images with a typical resolution of $\sim 35^{\prime \prime}$ to $40^{\prime \prime}$.

To properly image the diffuse cluster radio emission, it is necessary to subtract the contribution (to the flux and morphology) of the embedded discrete sources. Thanks to its configuration, which provides simultanously good coverage of short and long spacings in the $u-v$ plane, the GMRT is particularly suited to performing accurate source subtraction. Where necessary, we carefully subtracted the individual sources from the $u-v$ data, and then produced images of the diffuse cluster sources from the subtracted dataset. In particular, point-like sources with peak flux density $\geq 5-6 \sigma$ were subtracted from the diffuse emission in the $u-v$ plane. For careful subtraction of the extended radio galaxies we made an image cutting the innermost regions of the $u-v$ plane to avoid subtracting any possible contribution from the diffuse cluster emission, and tapering the visibilities for the best imaging of their extended emission. We then subtracted the clean components from the $u-v$ data and finally imaged the source subtracted dataset. For each source (point-like and extended), we ensured consistency between the total flux density subtracted as clean components from the $u-v$ data and the flux density measured in the full resolution image.

We are confident that any residual contribution of emission from extended sources to the flux and morphology of the diffuse cluster emission is negligible. The contribution of faint embedded sources whose flux density is just below our threshold is also negligible. Radio source counts show that the number of radio sources with mJy and sub-mJy flux density is $\sim 0.01$ for a sky region with an average largest angular size of $\sim 4-5^{\prime}$, as is the case for the cluster sources presented in this paper (e.g. Bondi et al. 2007; Venturi et al. 2002, and references therein).

Considering the uncertainties in the source subtraction procedure, we associate a final error of $8 \%$ to the flux density of the diffuse sources. 
Table 3. Properties of the discrete radio sources.

\begin{tabular}{|c|c|c|c|c|c|}
\hline $\begin{array}{l}\text { Cluster } \\
\text { name }\end{array}$ & Source & $\begin{array}{c}\mathrm{RA}^{a} \\
(\mathrm{~h}, \mathrm{~m}, \mathrm{~s})\end{array}$ & $\begin{array}{c}\operatorname{Dec}^{a} \\
\left({ }^{\operatorname{deg}},,^{\prime},{ }^{\prime \prime}\right)\end{array}$ & $\begin{array}{c}S_{\text {peak }, 325 \mathrm{MHz}} \\
\left(\mathrm{mJy} \mathrm{beam}^{-1}\right)\end{array}$ & $\begin{array}{c}\mathrm{S}_{\mathrm{tot}, 325 \mathrm{MHz}} \\
\text { (mJy) }\end{array}$ \\
\hline A 2744 & $\begin{array}{l}\text { S1 } \\
\text { S2 } \\
\text { S3 } \\
\text { S4 }\end{array}$ & $\begin{array}{llll}00 & 14 & 21.6 \\
00 & 14 & 33.8 \\
00 & 14 & 39.9 \\
00 & 14 & 44.5\end{array}$ & $\begin{array}{l}-302556 \\
-302231 \\
-302820 \\
-302620\end{array}$ & $\begin{array}{l}7.4 \pm 0.4 \\
6.3 \pm 0.3 \\
2.1 \pm 0.1 \\
9.8 \pm 0.5\end{array}$ & $\begin{array}{c}14.0 \pm 0.7 \\
7.5 \pm 0.4 \\
2.4 \pm 0.1 \\
83.6 \pm 4.2^{b}\end{array}$ \\
\hline A 209 & $\begin{array}{l}\text { S1 } \\
\text { S2 }\end{array}$ & $\begin{array}{l}013152.5 \\
013156.5\end{array}$ & $\begin{array}{l}-133700 \\
-133517\end{array}$ & $\begin{array}{c}40.9 \pm 2.0 \\
7.0 \pm 0.4\end{array}$ & $\begin{array}{l}81.7 \pm 4.1^{b} \\
13.2 \pm 0.7\end{array}$ \\
\hline Z2661 & $\begin{array}{l}\text { S1 } \\
\text { S2 } \\
\text { S3 } \\
\text { S4 }\end{array}$ & $\begin{array}{l}094947.1 \\
094949.1 \\
094950.3 \\
094952.5\end{array}$ & $\begin{array}{c}+170600 \\
+170657 \\
170634 \\
170734\end{array}$ & $\begin{array}{l}2.0 \pm 0.1 \\
3.0 \pm 0.2 \\
1.5 \pm 0.1 \\
6.2 \pm 0.3\end{array}$ & $\begin{array}{c}7.8 \pm 0.4^{b} \\
5.1 \pm 0.3 \\
3.2 \pm 0.2^{b} \\
8.7 \pm 0.4\end{array}$ \\
\hline A 1300 & $\begin{array}{l}\text { A1 } \\
\text { A2 } \\
\text { A2 } \\
\text { A3 } \\
\text { B1 }\end{array}$ & $\begin{array}{lll}11 & 3154.4 \\
11 & 3154.3 \\
& & \\
11 & 31 & 54.9 \\
11 & 31 & 43.7\end{array}$ & $\begin{array}{l}-195539 \\
-195355 \\
-195205 \\
-195255\end{array}$ & $\begin{array}{l}50.3 \pm 2.5 \\
92.5 \pm 4.6 \\
14.1 \pm 0.7 \\
19.6 \pm 1.0\end{array}$ & $\begin{array}{c}60.8 \pm 3.0 \\
197.8 \pm 9.9^{b} \\
216.1 \pm 10.8^{b, c} \\
23.1 \pm 1.2^{b} \\
40.7 \pm 2.0^{b}\end{array}$ \\
\hline A 1682 & $\begin{array}{l}\text { S1 } \\
\text { S2 } \\
\text { S3 }\end{array}$ & $\begin{array}{lll}13 & 06 & 45.6 \\
13 & 06 & 49.9 \\
13 & 07 & 03.7\end{array}$ & $\begin{array}{l}+463332 \\
+463333 \\
+463347\end{array}$ & $\begin{array}{c}400.0 \pm 20.0 \\
29.8 \pm 1.5 \\
5.6 \pm 0.3\end{array}$ & $\begin{array}{c}1131.6 \pm 56.6^{b, d} \\
50.3 \pm 2.5 \\
6.9 \pm 0.3\end{array}$ \\
\hline RXCJ 1314.4-2515 & $\begin{array}{l}\text { S1 } \\
\text { S2 }\end{array}$ & $\begin{array}{lll}13 & 14 & 30.4 \\
13 & 14 & 48.5\end{array}$ & $\begin{array}{lll}-25 & 1715 \\
-25 & 1617\end{array}$ & $\begin{array}{l}5.8 \pm 0.3 \\
7.9 \pm 0.4\end{array}$ & $\begin{array}{l}11.5 \pm 0.6 \\
18.0 \pm 0.9\end{array}$ \\
\hline A $1758 \mathrm{~N}$ & $\begin{array}{r}\mathrm{S} 1 \\
\mathrm{~S} 2 \\
\mathrm{~S} 3 \\
\mathrm{~S} 4^{a} \\
\mathrm{~S} 5\end{array}$ & $\begin{array}{lll}13 & 32 & 35.7 \\
13 & 32 & 39.0 \\
13 & 32 & 39.9 \\
13 & 32 & 54.8 \\
13 & 32 & 59.6\end{array}$ & $\begin{array}{l}+503235 \\
+503336 \\
+503432 \\
+503135 \\
+503122\end{array}$ & $\begin{array}{c}1.0 \pm 0.1 \\
16.9 \pm 0.8 \\
18.8 \pm 0.9 \\
145.3 \pm 7.3 \\
18.0 \pm 9.0\end{array}$ & $\begin{array}{c}2.0 \pm 0.1 \\
21.0 \pm 1.1 \\
27.2 \pm 1.4 \\
514.6 \pm 25.7 \\
40.3 \pm 2.0\end{array}$ \\
\hline
\end{tabular}

Notes. ${ }^{(a)}$ Coordinates of the radio peak; ${ }^{(b)}$ measured using TVSTAT; ${ }^{(c)}$ the value in brackets includes the bridge between A1 and A2; ${ }^{(d)}$ this value includes the E tail.

\section{Diffuse cluster sources at low frequency}

For each cluster presented in this section, Table 3 provides the position and flux density of the individual radio sources embedded in the diffuse emission, as measured on the full resolution images with the AIPS task JMFIT for unresolved sources and TVSTAT for extended sources. Table 4 summarises the observational properties of the diffuse emission in each cluster (the morphological classification is given in Col. 2; the total flux density and linear size are reported in Cols. 4 and 5, respectively) imaged after subtraction of the discrete radio galaxies listed in Table 3. The bottom part of Table 4 reports the information for the clusters presented in separate papers. In Table 5 we list the candidate diffuse cluster sources that still need to be confirmed.

\subsection{Clusters with a giant radio halo}

\subsubsection{A 209}

A $209(z=0.206)$ is a massive merging cluster with $L_{\mathrm{X},[0.1-2.4 \mathrm{keV}]}=6.3 \times 10^{44} \mathrm{erg} \mathrm{s}^{-1}$ (see V07 for a review of its properties). It hosts a giant radio halo, imaged with the GMRT at $610 \mathrm{MHz}$ (V07) and with the VLA at $1.4 \mathrm{GHz}$ (Giovannini et al. 2009; Giacintucci et al. 2013, hereinafter G13).

The $325 \mathrm{MHz}$ data were affected by strong interference, which required massive data editing and prevented high quality imaging of the diffuse cluster emission: no individual source subtraction was performed for this cluster. Our images, presented in Fig. 1, are in agreement with those at $610 \mathrm{MHz}$, both in shape and extent (Fig. 2 in V07). The radio halo covers only the most luminous part of the X-ray emission, and is smaller than imaged at $1.4 \mathrm{GHz}$, most likely as a consequence of the RFI removal editing, which forced us to drop many short spacings. The flux density of the radio halo is $S_{325 \mathrm{MHz}}=74 \pm 6 \mathrm{mJy}$, measured from the image shown in the right-hand panel of Fig. 1 after subtraction of the flux density of the discrete radio sources S1 and $\mathrm{S} 2$ reported in Table 3 (Fig. 1 left). We consider this value and the largest linear size measured from the right-hand panel of Fig. 1 (Table 4) as lower limits. V07 reported a flux density $S_{610 \mathrm{MHz}}=24.0 \pm 3.6$, Giovannini et al. (2009) report a value at $1.4 \mathrm{GHz}$ of $S_{1.4 \mathrm{GHz}}=16.9 \mathrm{mJy}$, while a re-analysis of the archival VLA $1.4 \mathrm{GHz}$ data performed in G13 led to a flux density estimate measurement $S_{1.4 \mathrm{GHz}}=15.0 \pm 0.7 \mathrm{mJy}$. Considering that the flux density of the radio halo at $610 \mathrm{MHz}$ is most likely understimated (as discussed in V08), and in light of the limited quality of the $325 \mathrm{MHz}$ data, we could not perform a spectral study for this cluster and did not include A 209 in the discussion carried out in Sect. 4.4.1.

\subsection{2. $A 1758 \mathrm{~N}$}

A $1758 \mathrm{~N}\left(z=0.2782\right.$, Boschin et al. 2012; $L_{\mathrm{X},[0.1-2.4 \mathrm{KeV}]}=$ $\left.12.3 \times 10^{44} \mathrm{erg} \mathrm{s}^{-1}\right)$ is part of a well-known pair of two merging clusters, A $1758 \mathrm{~N}$ and A $1758 \mathrm{~S}$, separated by $\sim 8^{\prime}$ in the plane of the sky and $\sim 2100 \mathrm{~km} \mathrm{~s}^{-1}$ in the velocity space, both formed 
Table 4. Properties of the diffuse cluster sources in the GMRT radio Halo survey.

\begin{tabular}{lcccccc}
\hline \hline Cluster name & Radio source & $\begin{array}{c}v \\
(\mathrm{MHz})\end{array}$ & $\begin{array}{c}S_{v} \\
(\mathrm{mJy})\end{array}$ & $\begin{array}{c}P_{v} \\
\mathrm{~W} \mathrm{HZ}^{-1}\end{array}$ & $\begin{array}{c}\text { LLS } \\
(\mathrm{Mpc})\end{array}$ & $\begin{array}{c}\text { HPBW } \\
\left({ }^{\prime \prime} \times \times^{\prime \prime}\right)\end{array}$ \\
\hline A 2744 & GRH & 325 & $323 \pm 26$ & $9.68 \times 10^{25}$ & $\sim 1.9$ & $35.0 \times 35.0$ \\
& Rel & 325 & $122 \pm 10$ & $3.66 \times 10^{25}$ & $\sim 1.3$ & $35.0 \times 35.0$ \\
A 209 & GRH & 325 & $74 \pm 6^{a}$ & $8.91 \times 10^{24}$ & $\sim 0.6^{a}$ & $25.0 \times 25.0$ \\
A 1300 & GRH & 325 & $130 \pm 10$ & $3.92 \times 10^{25}$ & $\sim 0.9$ & $28.0 \times 28.0$ \\
& Rel & 325 & $75 \pm 6$ & $2.26 \times 10^{25}$ & $\sim 0.5$ & $28.0 \times 28.0$ \\
& Cand. Rel & 325 & $23 \pm 2$ & $6.94 \times 10^{24}$ & $\sim 0.7$ & $28.0 \times 28.0$ \\
A 1682 & Diffuse Comp. & 240 & $46 \pm 4$ & $6.82 \times 10^{24}$ & $\sim 0.3$ & $18.3 \times 14.0$ \\
RXCJ 1314.4-2515 & W Rel & 325 & $137 \pm 11$ & $2.42 \times 10^{25}$ & & $26.1 \times 10.2$ \\
& E Rel & 325 & $52 \pm 4$ & $9.18 \times 10^{24}$ & & $26.1 \times 10.2$ \\
& RH & 325 & $40 \pm 3$ & $7.06 \times 10^{24}$ & & $26.1 \times 10.2$ \\
A 1758N & GRH & 325 & $155 \pm 12$ & $3.75 \times 10^{25}$ & $\sim 1.5$ & $35.0 \times 35.0$ \\
\hline A 521 & GRH & 240 & $152 \pm 15$ & $2.79 \times 10^{25}$ & $\sim 0.9$ & $35.0 \times 35.0$ \\
A 521 & Rel & 240 & $180 \pm 10$ & $3.30 \times 10^{25}$ & $\sim 1$ & $35.0 \times 35.0$ \\
A 521 & Rel & 325 & $114 \pm 6$ & $2.09 \times 10^{25}$ & $\sim 1$ & $16.0 \times 13.0$ \\
A 697 & GRH & 325 & $47.3 \pm 2.7$ & $1.16 \times 10^{25}$ & $\sim 1.3$ & $46.8 \times 41.4$ \\
A 781 & Rel & 325 & $93.3 \pm 7.5$ & $2.61 \times 10^{25}$ & $\sim 0.8$ & $40.0 \times 37.0$ \\
RXCJ 2003.5-2323 $^{f}$ & GRH & 240 & $360 \pm 18$ & $1.16 \times 10^{26}$ & $\sim 1.4$ & $35.0 \times 35.0$ \\
\hline
\end{tabular}

Notes. ${ }^{(a)}$ The value should be considered a lower limit, see Sect. 3.1.1. ${ }^{(b)}$ Information taken from Brunetti et al. (2008). ${ }^{(c)}$ Information taken from Giacintucci et al. (2008). ${ }^{(d)}$ Information taken from Macario et al. (2010). ${ }^{(e)}$ Information taken from Venturi et al. (2011a). ${ }^{(f)}$ Information taken from Giacintucci et al. (2009).

Table 5. Candidate diffuse cluster emission.

\begin{tabular}{lccc}
\hline \hline Cluster name & Candidate source & $\begin{array}{c}v \\
(\mathrm{MHz})\end{array}$ & $\begin{array}{c}S_{v} \\
(\mathrm{mJy})\end{array}$ \\
\hline $\mathrm{A} \mathrm{781}$ & & 325 & $\sim 20-30$ \\
$\mathrm{~A} \mathrm{1682}$ & USSRH? & 240 & $\sim 80$ \\
$\mathrm{Z}^{b} 661^{c}$ & GRH? & 610 & $\sim 5.9$ \\
\hline
\end{tabular}

Notes. ${ }^{(a)}$ From Venturi et al. (2011a). ${ }^{(b)}$ From Venturi et al. (2011b). (c) From V08.

by two merging clusters (Ragozzine et al. 2012). A detailed Chandra and XMM-Newton X-ray study (David \& Kempner 2004) reveals that both clusters are characterised by very complex X-ray morphology, with several clumps of emission, as commonly found in cases of ongoing cluster merging activity. A $1758 \mathrm{~N}$ shows more extreme X-ray properties, with higher $\mathrm{X}$-ray luminosity and temperature compared to A 1758 S. Its total mass is $\sim 2-3 \times 10^{15} M_{\odot}$, and a new optical analysis confirms that it is a recent merger (Boschin et al. 2012; Ragozzine et al. 2012). A $1758 \mathrm{~N}$ is also much more interesting in the radio band, with a well-known central narrow angle tail radio galaxy (O'Dea \& Owen 1985) and diffuse central emission visible both on the Northern VLA Sky Survey (NVSS) and on the WEsterbork Northern Sky Survey (WENSS), as reported in Kempner \& Sarazin (2001). VLA observations at $1.4 \mathrm{GHz}$ have recently been presented in Giovannini et al. (2009) and reanalysed in G13.

The images of A $1758 \mathrm{~N}$ are shown in Fig. 2. Their quality is one of the best in our GMRT $325 \mathrm{MHz}$ atlas (see Table 2). The left-hand panel shows the total radio emission from the cluster central region imaged at full resolution overlaid on the optical image. The right-hand panel shows the overlay between the Chandra X-ray image and the diffuse emission, after subtraction of the discrete point-like and extended sources S1 to S5 from the $u-v$ data (see Sect. 2.2, Fig. 2 left-hand panel, and Table 3 ). The diffuse radio emission covers only a portion of the
X-ray brightness distribution. It has a filamentary structure, and is aligned in the north-west/south-east direction. The radio and $\mathrm{X}$-ray peaks of emission are in good spatial coincidence in the north-western part of the cluster, while a misplacement is clear in the south-eastern cluster region.

The total flux density of the diffuse emission, measured within the $3 \sigma$ contour, is $S_{325 \mathrm{MHz}}=155 \pm 12 \mathrm{mJy}$. The total size of this structure is $\sim 1.5 \mathrm{Mpc}$. The overall shape and size of the diffuse emission detected at $325 \mathrm{MHz}$ is in reasonable agreement with the re-analysis carried out in G13. Giovannini et al. (2009) classify the radio emission as a radio halo and a double relic. Indeed the morphology of this source is very complex, however we find it difficult to disentangle possible different components. The overall spatial coincidence between the radio and $\mathrm{X}$-ray emission, as is clear from the right-hand panel of Fig. 2, suggests that the source is centrally located, so we consider it a giant radio halo.

The spectral index of the whole emission, using the total $1.4 \mathrm{GHz}$ flux density reported in $\mathrm{G} 13\left(S_{1.4 \mathrm{GHz}}=23 \pm 5 \mathrm{mJy}\right.$, integrated over the same area) is $\alpha_{325 \mathrm{MHz}}^{1.4 \mathrm{GHz}}=1.31 \pm 0.16$.

\subsection{Clusters with halo and relics}

Three clusters in the sample host multiple diffuse cluster sources. A 1300 and A 2744, both located at $z \sim 0.3$ and with similar $\mathrm{X}$-ray luminosity, host a giant radio halo and a relic. They belong to the GMRT radio halo cluster sample, but because they are known radio haloes they were not observed at $610 \mathrm{MHz}$ in our earlier works (V07 \& V08). RXCJ 1314.5-2515 hosts spectacular diffuse emission in the shape of a radio halo and two very long and symmetric relics.

\subsubsection{A 1300}

A 1300 is located at $z=0.308$ and has $L_{X,[0.1-2.4 \mathrm{KeV}]}=$ $1.4 \times 10^{45} \mathrm{erg} \mathrm{s}^{-1}$. It hosts a radio halo, first reported in Reid et al. (1999, hereinafter R99) on the basis of radio observations carried out with the Molonglo Observatory Synthesis Telescope 

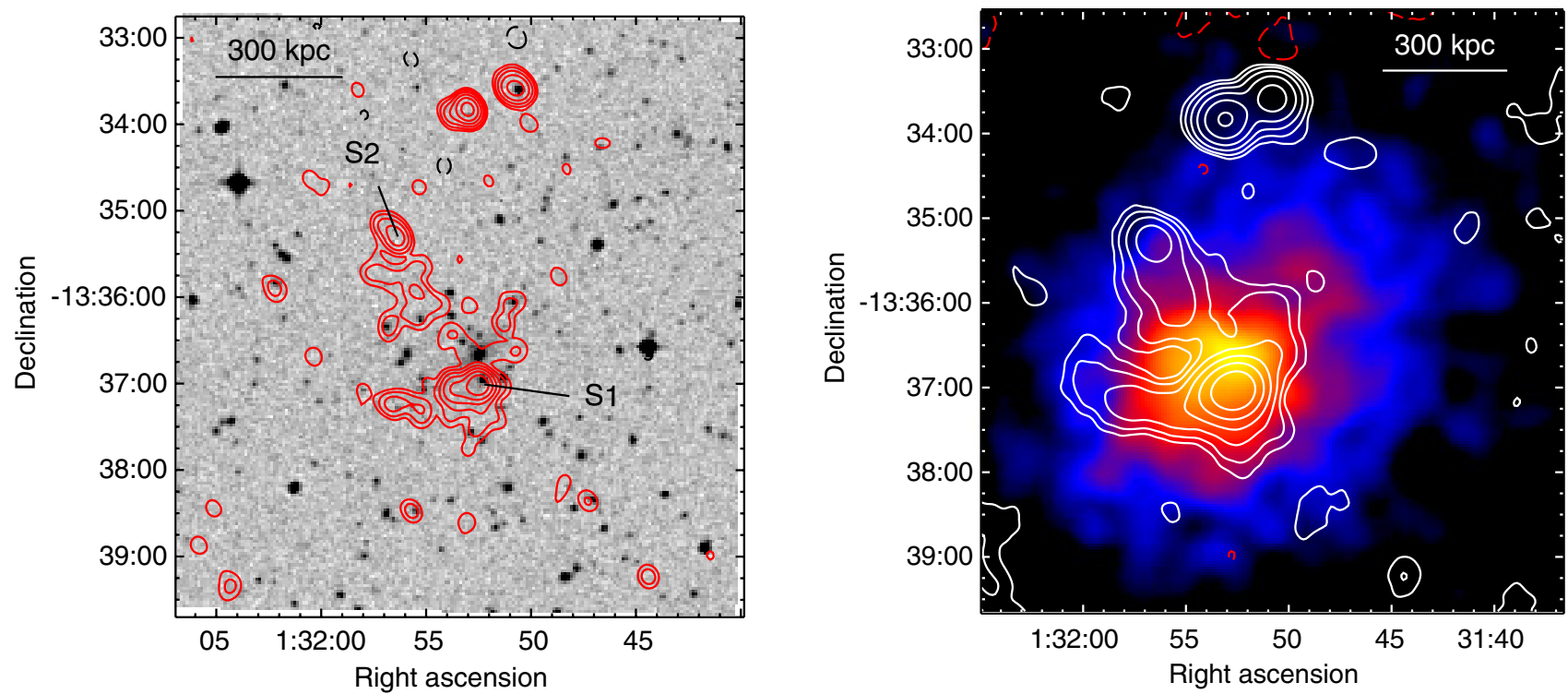

Fig. 1. Left: GMRT $325 \mathrm{MHz}$ radio contours of A 209 at full resolution $\left(11.0^{\prime \prime} \times 8.7^{\prime \prime}\right.$, p.a. $\left.30^{\circ}\right)$, overlaid on the optical POSS-2 image. The $1 \sigma$ level in the radio image is $0.12 \mathrm{mJy}_{\text {beam }}^{-1}$ and red contours are spaced by a factor 2 starting from $+3 \sigma=0.36 \mathrm{mJy} \mathrm{beam}^{-1}$. Black dashed contours correspond to the $-3 \sigma$ level. Labels S1 and S2 show are the radio galaxies embedded in the radio halo emission. Right: GMRT $325 \mathrm{MHz}$ radio contours at the resolution of $25.0^{\prime \prime} \times 25.0^{\prime \prime}$, p.a. $0^{\circ}$, superposed to the smoothed Chandra X-ray image. No source subtraction has been performed. White contours start at $+3 \sigma=0.9 \mathrm{mJy}^{-1}$ beam $^{-1}$ and then scale by a factor 2 . The $-3 \sigma$ level is shown as red dashed contours.
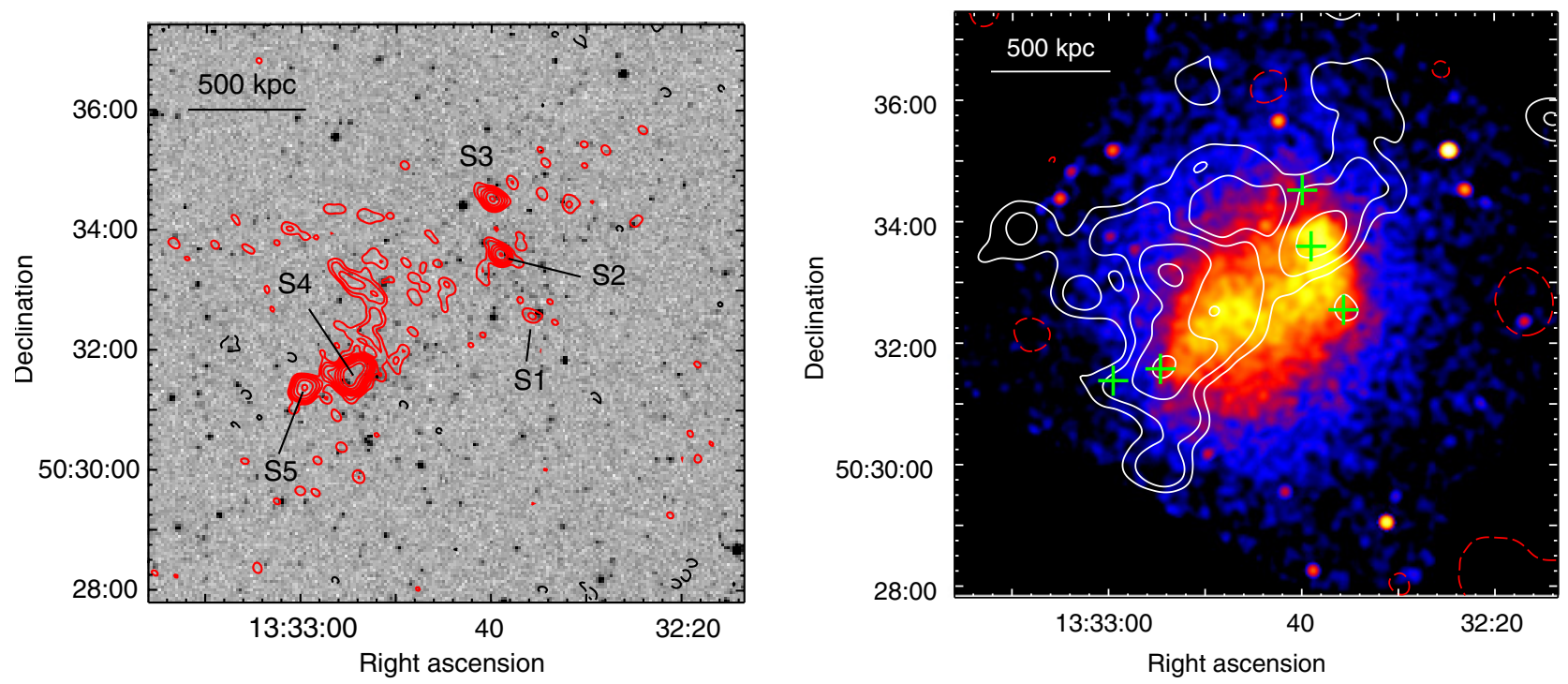

Fig. 2. Left: GMRT $325 \mathrm{MHz}$ radio contours of the emission from A $1758 \mathrm{~N}$ at the full resolution $\left(10.5^{\prime \prime} \times 8.4^{\prime \prime}\right.$, p.a. $\left.58^{\circ}\right)$, overlaid on the POSS-2 red image. The $1 \sigma$ noise level is $0.1 \mathrm{mJy}$ beam $^{-1}$. Red contours start at $0.4 \mathrm{mJy}_{\text {beam }}{ }^{-1}$ and scale by a factor of 2 . Black dashed contours correspond to the $-3 \sigma$ level. Individual radio galaxies are labelled from S1 to S5. Right: $325 \mathrm{MHz}$ contours of the giant radio halo overlaid on the smoothed Chandra X-ray image. The radio image has been obtained after subtracting the discrete embedded sources (S1 to S5), whose position is marked as green crosses. The restoring beam is $35.0^{\prime \prime} \times 35.0^{\prime \prime}$, p.a. $0^{\circ}$. The $1 \sigma$ is $0.4 \mathrm{mJy}_{\text {beam }}^{-1}$. White contours start at $+3 \sigma=0.12 \mathrm{mJy}^{\circ}$ beam ${ }^{-1}$ and scale by a factor 2 . The $-3 \sigma$ level is shown as red dashed contours.

(MOST) at $843 \mathrm{MHz}$ and with the Australia Telescope Compact Array (ATCA) from $1.34 \mathrm{GHz}$ to $8.6 \mathrm{GHz}$. With a temperature of $\sim 11 \mathrm{keV}$ (Pierre et al. 1997), A 1300 is one of the hottest clusters known. Optical and X-ray analysis (Pierre et al. 1997; Lemonon et al. 1997; Ziparo et al. 2012) suggests that it is a post-merger system. The radio observations by R99 show that A 1300 is very active in the radio band. Beyond the radio halo, a relic is located in the south-western periphery of the cluster, and a number of radio galaxies with extended emission are found at the cluster centre. In a recent optical and X-ray analysis, Ziparo et al. (2012) report on a possible shock front (consistent with a
Mach number $M=1.2 \pm 0.1$ ) and on a sharp edge in the galaxy distribution at the location of the relic.

Owing to strong interference, only the upper side band of the GMRT $325 \mathrm{MHz}$ dataset could be used in our imaging process. Figure 3 (left) shows the radio emission from the cluster centre (contours) at the intermediate resolution of $26.7^{\prime \prime} \times 18.1^{\prime \prime}$, overlaid on the full resolution image $\left(14.0^{\prime \prime} \times 8.8^{\prime \prime}\right)$, displayed in grey scale to highlight the position and morphology of the radio galaxies embedded in the diffuse cluster emission. The individual radio sources are labelled following the notation in R99 and their flux density is reported in Table 3. An elongated, thin 
T. Venturi et al.: Low frequency follow up of the GMRT radio Halo survey
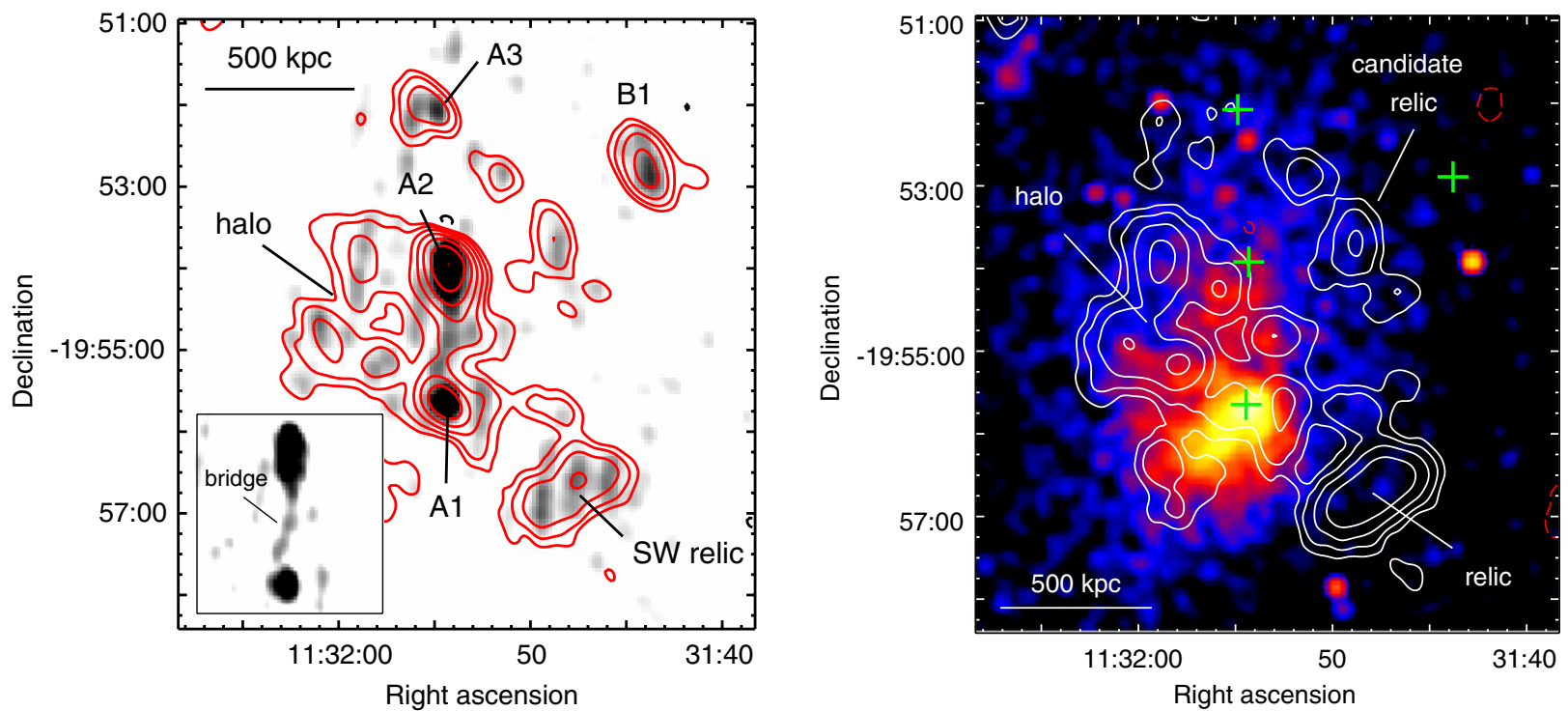

Fig. 3. Left: GMRT $325 \mathrm{MHz}$ radio contours of the emission from A 1300 at the resolution of $27.6^{\prime \prime} \times 18.1^{\prime \prime}$, p.a. $43^{\circ}$, overlaid on the grey-scale full-resolution image $\left(14.0^{\prime \prime} \times 8.8^{\prime \prime}\right.$, p.a. $\left.8^{\circ}\right)$. The $1 \sigma$ noise level of the contour image is $0.6 \mathrm{mJy} \mathrm{beam}^{-1}$. Red contours start at $1.8 \mathrm{mJy} \mathrm{b}^{-1}$ and then scale by a factor of 2 . The $1 \sigma$ noise level of the grey scale image is $0.45 \mathrm{mJy}_{\text {beam }}^{-1}$. Individual radio galaxies are labelled following the notation in R99. Right: radio contours of the central cluster region after subtracting the discrete radio sources (the green crosses mark their location). The restoring beam is $28.0^{\prime \prime} \times 28.0^{\prime \prime}$, p.a. $0^{\circ}$. The $1 \sigma$ is $0.5 \mathrm{mJy}$ beam ${ }^{-1}$. White contours start at $1.5 \mathrm{mJy} \mathrm{beam}^{-1}$ and then scale by a factor of 2 . The $-3 \sigma$ level is shown as red dashed contours.

structure, hereinafter referred to as bridge (see inset in Fig. 3) connects the head-tail radio galaxy A2 to the point source A1. It is not clear whether such a feature is an extension of the tail of A2 or a brightness peak of the diffuse radio halo. For this reason, Table 3 reports the flux density of A2 with and without the contribution of the bridge, which accounts for $\sim 18 \mathrm{mJy}$. The radio sources labelled B2, B3, and 12 in R99 are undetected in our $325 \mathrm{MHz}$ images, most likely due to their spectral slope. We subtracted the components of the individual radio galaxies (Table 3 ) from the $u-v$ data (we considered the bridge as part of A2 and we subtracted it as well) and produced a low resolution image of the residual diffuse emission, which is shown in the right-hand panel of Fig. 3, overlaid on the smoothed Chandra X-ray image. The $325 \mathrm{MHz}$ brightness distribution of the radio halo is fairly uniform.

The shape of the radio halo at this frequency is similar to the higher frequency images, with the diffuse emission extending mainly east of the two radio sources A1 and A2. The overall size is also comparable to the one reported in R99, with a largest linear size (LAS) of 3.3' (largest linear size LLS $~ 890 \mathrm{kpc}$ ). The south-western ( $\mathrm{S}-\mathrm{W}$ hereinafter) relic (B3 in R99), too, is similar in shape, extent and brightness distribution to the earlier images, and its LLS at $325 \mathrm{MHz}$ is $\sim 450 \mathrm{kpc}$. Our image shows the existence of an arc-shaped elongated feature, not visible in any of the images published in R99, located north-west of the cluster centre, which accounts for $S_{325 \mathrm{MHz}}=23 \pm 2 \mathrm{mJy}$ and is $\sim 700 \mathrm{kpc}$ in extent. The overall morphology and location suggest that it might be another relic (see Fig. 3 right-hand panel).

The total flux density measured in the radio halo, integrating within the $3 \sigma$ contour level in Fig. 3 (right), is $S_{325} \mathrm{MHz}=130 \pm$ $10 \mathrm{mJy}$ (the flux density of the bridge was not included). The flux density of the S-W relic is $S_{325} \mathrm{MHz}=75 \pm 6 \mathrm{mJy}$.

We combined our flux density values with those reported by $\mathrm{R} 99$ in the frequency range $843 \mathrm{MHz}-2.4 \mathrm{GHz}$ to provide an estimate of the spectral index for the halo and the $\mathrm{SW}$ relic. The $1.36 \mathrm{GHz}$ flux density of the radio halo reported in R99 is 10 mJy (consistent with the value we measured on the NVSS after subtraction of the contribution of the two embedded sources A1 and A2), which provides a spectral index $\alpha_{325 \mathrm{MHz}}^{1.4 \mathrm{GHz}}=1.8$. Including the bridge in the $325 \mathrm{MHz}$ emission from the halo would further steepen the spectrum (up to $\alpha=1.9$ ). The $\mathrm{S}-\mathrm{W}$ relic has $\alpha_{325 \mathrm{MHz}}^{843 \mathrm{MHz}}=0.86$, which steepens to $\alpha_{1.34 \mathrm{GHz}}^{2.4 \mathrm{GHz}}=1.3$. We are aware that it is impossible to account for the different $u-v$ coverages of the ATCA, MOST, GMRT, and VLA. For this reason we regard these spectral considerations as indicative, and we do not include this cluster in the discussion performed in Sect. 4.4.1.

The overlay in the right-hand panel of Fig. 3 shows that the radio halo extends mainly north-east of the peak in the X-ray surface brightness. Both the $\mathrm{S}-\mathrm{W}$ and the candidate relics are located on the border of the detected X-ray emission.

\subsubsection{A 2744}

A 2744 is located at the redshift $z=0.3066$ and has $L_{\mathrm{X},[0.1-2.4 \mathrm{KeV}]}=1.2 \times 10^{45} \mathrm{erg} \mathrm{s}^{-1}$. The cluster hosts a well known spectacular diffuse radio emission, with a giant radio halo and a giant relic. VLA radio observations at $1.4 \mathrm{GHz}$ are reported in Govoni et al. (2001) and 327 MHz in Orrú et al. (2007), respectively.

The cluster is a complex galaxy merger and has been studied in detail in a wide range of energy bands. Chandra observations (Kempner \& David 2004) show that the cluster is formed by a main irregular component and a smaller subcluster to the northwest. The main cluster shows a very complex substructure with a strong peak and a number of "ridges" in different directions. The subcluster is connected to the main one by a bridge of fainter $\mathrm{X}$-ray emission, and its brigthtness distribution suggests that it is moving towards the main condensation (Kempner \& David 2004). The very unrelaxed dynamical state of A 2744 is confirmed by optical spectroscopy, which reveals a redshift bimodal distribution and an unusually high merger velocity (Girardi \& Mezzetti 2001). Merten et al. (2011) recently concluded that the main cluster and the subcluster are both post-merger systems, 

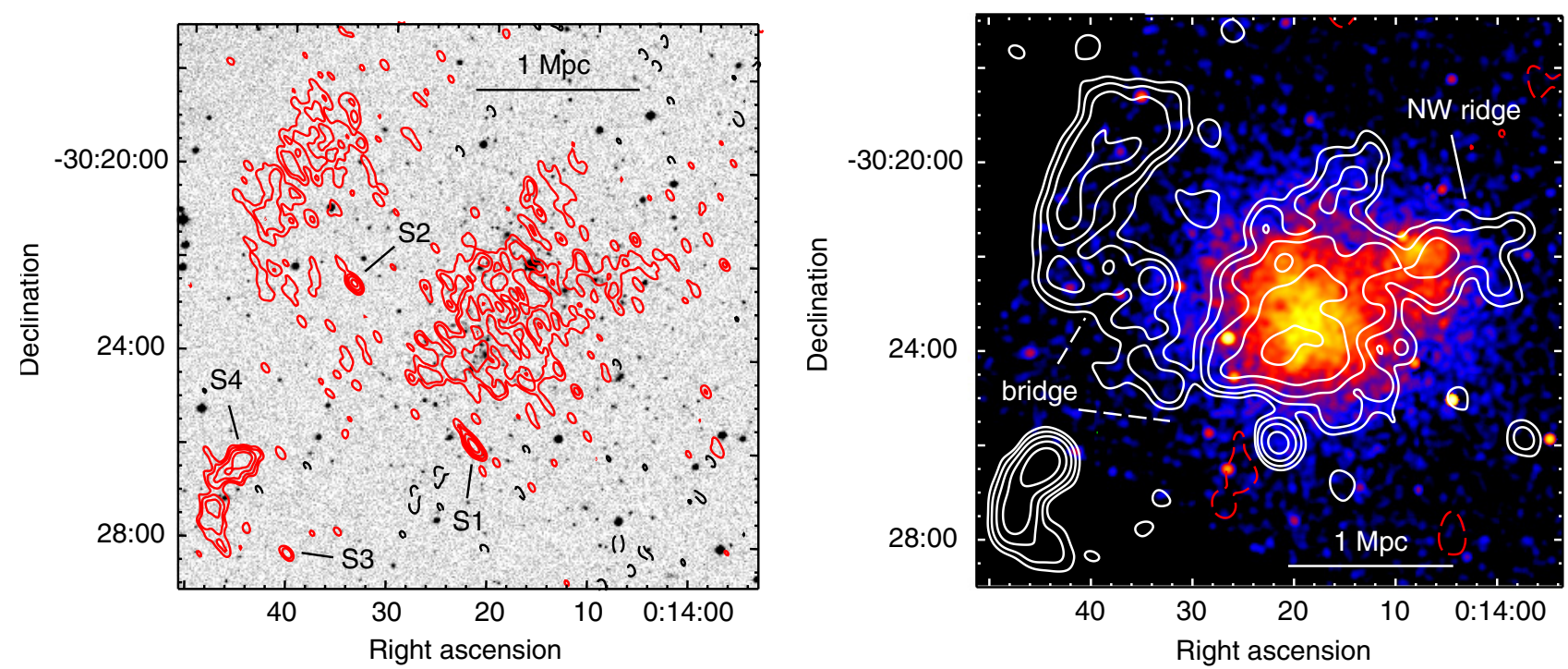

Fig. 4. Left: GMRT $325 \mathrm{MHz}$ radio contours of A 2744 at the full resolution of $15.9^{\prime \prime} \times 8.5^{\prime \prime}$, p.a. $38^{\circ}$, overlaid on the POSS-2 red image. The $1 \sigma$ noise level is $0.15 \mathrm{mJy}_{\text {beam }}^{-1}$. Red contours start at $0.5 \mathrm{mJy} \mathrm{beam}^{-1}$ and then scale by a factor of 2 . Dashed, black contours correspond to the $-3 \sigma$ level. Individual radio galaxies are labelled from S1 to S4. Right: low resolution $\left(35.0^{\prime \prime} \times 35.0^{\prime \prime}\right.$, p.a. $\left.0^{\circ}\right)$ radio contours overlaid on the smoothed Chandra X-ray image. The $1 \sigma$ noise level in the radio image is $0.35 \mathrm{mJy}$ beam $^{-1}$. White contours start at $1 \mathrm{mJy}^{\text {beam }}{ }^{-1}$ and then scale by a factor of 2 . The $-3 \sigma$ level is shown as dashed, red contours.

and show properties similar to the Bullet cluster, with a misplacement between the gas and the dark matter.

Figure 4 shows our $325 \mathrm{MHz}$ GMRT images. Both the radio halo and the relic are already visible in the full resolution image (left-hand panel). The two sources are connected by a faint bridge (right-hand panel), visible also in the VLA $1.4 \mathrm{GHz}$ image in Govoni et al. (2001). Thanks to the better sensitivity ( a factor of 3) of our image than those in Orrú et al. (2007), the radio halo in A 2744 is larger than previously imaged at $325 \mathrm{MHz}$. In particular, we detect a north-western ridge, labelled in the right-hand panel of Fig. 4, along the same direction of the subcluster X-ray emission. The largest linear size of the diffuse sources from the low resolution image (right-hand panel) is LLS $\sim 1.9 \mathrm{Mpc}$ for the halo (including the NW ridge) and LLS $\sim 1.3 \mathrm{Mpc}$ for the relic. The bridge extends for $\sim 700 \mathrm{kpc}$ between the halo and relic.

The radio/X-ray overlay in Fig. 4 (right) clearly shows that the emission from the radio halo extends over the whole X-ray emission and beyond, with a positional shift between the X-ray and radio peaks both for the main cluster and for the northwestern subcluster. The radio brightness distribution is asymmetrically peaked, and the southern part of the radio halo is edge-sharpened. It is noteworthy that Markevitch (2010) has recently reported on the detection of a X-ray surface brightness edge, suggesting the presence of a shock front, at the location of this radio edge-sharpening. The front has been confirmed by the Chandra analysis presented by Owers et al. (2012), who derived a Mach number of $M=1.4$ for the shock (see Fig. 12 in Markevitch 2010 for a radio/X-ray overlay of the shock region). Similar radio/X-ray spatial correlations have been observed in a few other clusters with a giant radio halo, e.g., A 520 (Markevitch et al. 2005) and A 754 (Macario et al. 2011a).

As is commonly found, the relic has a higher surface brightness compared to the halo, and its size agrees with the literature images. The radio/X-ray overlay confirms that the relic is located just outside the region where significant $\mathrm{X}$-ray emission is detected (see also Govoni et al. 2001).
From the low resolution image we measured a total flux density $S_{325 \mathrm{MHz}}=323 \pm 26 \mathrm{mJy}$ for the halo (excluding the bridge and source $\mathrm{S} 1$ ), and $S_{325 \mathrm{MHz}}=122 \pm 10 \mathrm{mJy}$ for the relic (excluding the bridge and source $\mathrm{S} 2$ ). The flux density in the bridge is $\sim 30 \mathrm{mJy}$. The measurements were taken within the $3 \sigma$ contour level. Both values are considerably higher than those reported in Orrú et al. (2007).

A 2744 is included in the re-analysis carried out in G13. Using their $1.4 \mathrm{GHz}$ flux density values over the same area, i.e. $S_{1.4 \mathrm{GHz}}=57 \pm 3$ and $20 \pm 1 \mathrm{mJy}$, respectively, for the halo and for the relic, we obtain $\alpha=1.19_{-0.11}^{+0.08}$ for the halo, and $\alpha=1.24 \pm 0.10$ for the relic.

\subsubsection{RXCJ 1314.4-2515}

The cluster RXCJ 1314.4-2515 $\left(z=0.244, L_{X,[0.1-2.4 \mathrm{KeV}]}=\right.$ $1.1 \times 10^{45} \mathrm{erg} \mathrm{s}^{-1}$ ) is a spectacular example of a major merger with a massive subcluster (Valtchanov et al. 2002). It was the first cluster where a radio halo and two relics were discovered (Feretti et al. 2005, V07). So far only two clusters with such features have been found (CIZA J 2242.8+5301, van Weeren et al. 2011b; MACS J 1752.0+4440, van Weeren et al. 2012). The two relics are elongated, Mpc-size structures located in opposite directions with respect to the cluster centre. Deep XMM-Newton observations led to the discovery of a prominent shock front, with Mach number $M \sim 2.5$, remarkably coincident with the outer border of the western relic and likely produced by the merger of the cluster with a massive subcluster (Mazzotta et al. 2011).

Our GMRT $325 \mathrm{MHz}$ observations were affected by a number of antenna failures and strong interference, nevertheless we managed to image the brightest features of the diffuse emission. The $325 \mathrm{MHz}$ cluster radio emission is reported in Fig. 5, where the full resolution image is overlaid on the optical frame (left) and a tapered image $\left(26^{\prime \prime} \times 10^{\prime \prime}\right)$ is overlaid on the smoothed XMM-Newton image (right).

Owing to the poor quality of our dataset, both relics are considerably smaller in size than the $610 \mathrm{MHz}$ images published in 
T. Venturi et al.: Low frequency follow up of the GMRT radio Halo survey
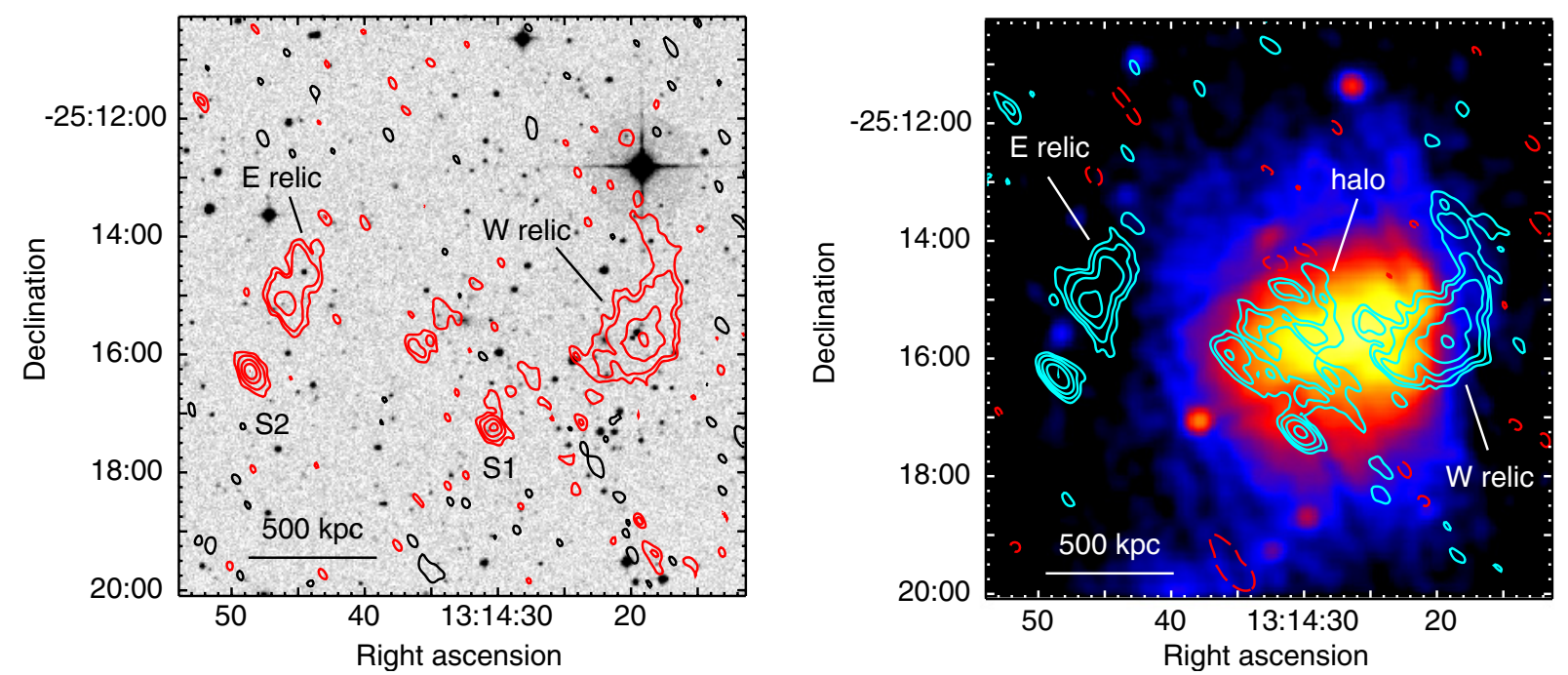

Fig. 5. Left: GMRT $325 \mathrm{MHz}$ image of RXCJ 1314.4-2515 at the resolution of $15.1^{\prime \prime} \times 8.0^{\prime \prime}$, p.a. $32^{\circ}$, overlaid on the POSS -2 red image. The $1 \sigma$ noise level is $0.16 \mathrm{mJy}^{-1}$ beam $^{-1}$. Red contours start at $0.5 \mathrm{mJy}^{-1}$ beam $^{-1}$ and then scale by a factor of 2 . Black contours correspond to the $-3 \sigma$ level. Individual radio galaxies are labelled S1 and S2. Right: GMRT $325 \mathrm{MHz}$ radio contours overlaid on the smoothed XMM-Newton X-ray image. The resolution of the radio image is $26.1^{\prime \prime} \times 10.2^{\prime \prime}$, p.a. $43^{\circ}$. The $1 \sigma$ noise level in the radio image is $\sim 0.2 \mathrm{mJy}$ beam ${ }^{-1}$. Cyan contours start at $0.6 \mathrm{mJy}_{\text {beam }}{ }^{-1}$ and then scale by a factor of 2 . The $-3 \sigma$ level is shown as red dashed contours.
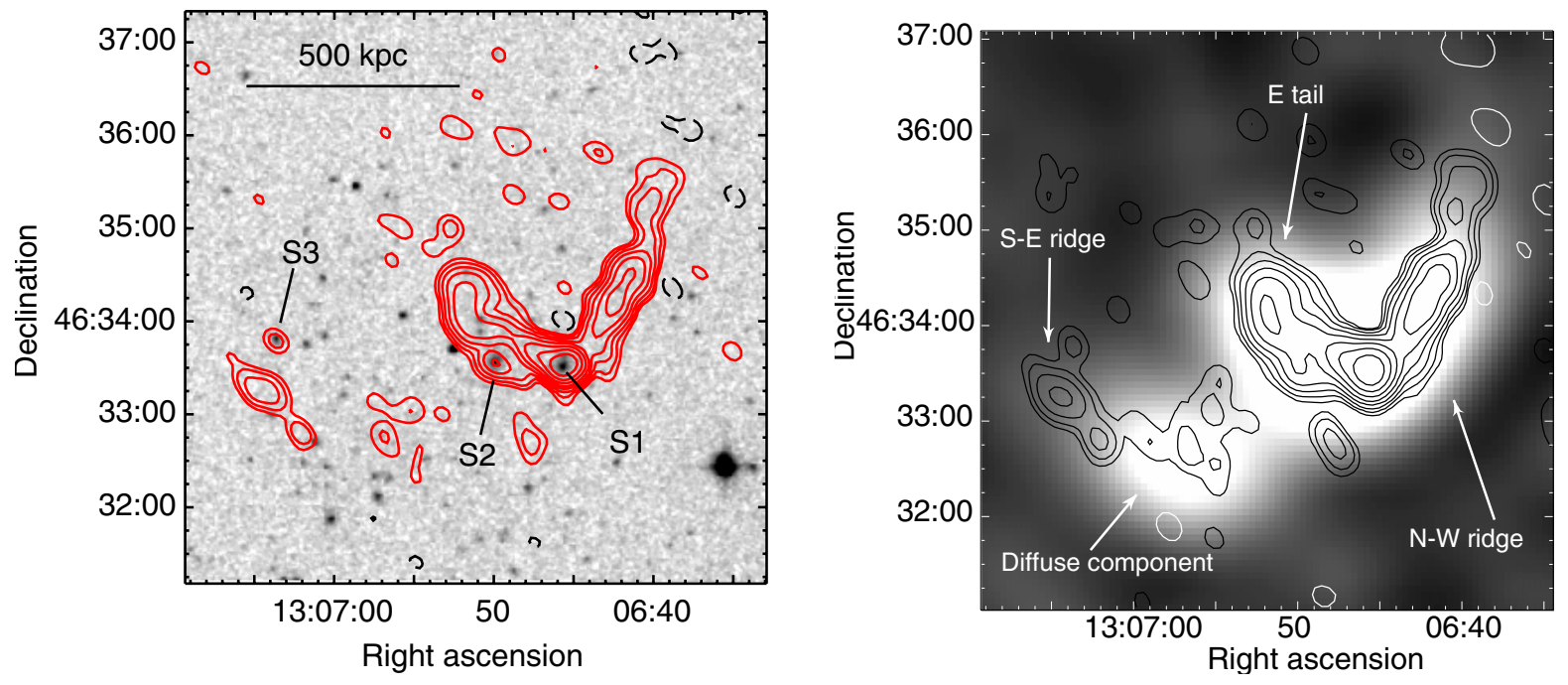

Fig. 6. Left: GMRT $240 \mathrm{MHz}$ image of A 1682 at the resolution of $12.5^{\prime \prime} \times 9.2^{\prime \prime}$, p.a. $55.7^{\circ}$. The $1 \sigma \mathrm{rms}$ in the image is $0.6 \mathrm{mJy}^{-1}$ beam ${ }^{-1}$. Red contours start at $2 \mathrm{mJy}_{\text {beam }}{ }^{-1}$ and scale by a factor of 2 . Black dashed contours correspond to the $-2 \mathrm{mJy}$ beam ${ }^{-1}$ level. Individual radio galaxies are labelled S1, S2 and S3. The optical image is the red plate of the Digitized Sky Survey (DSS-2). Right: GMRT $240 \mathrm{MHz}$ contours overlaid on the VLSS $74 \mathrm{MHz}$ emission (grey scale). The resolution of the $240 \mathrm{MHz}$ image is $18.3^{\prime \prime} \times 14.0^{\prime \prime}$, p.a. $21.7^{\circ}$. Black contours start at $2 \mathrm{mJy}$ beam ${ }^{-1}$ $\left(1 \sigma=0.6 \mathrm{mJy}\right.$ beam $\left.^{-1}\right)$ and are spaced by a factor of 2 . White contours correspond to the $-2 \mathrm{mJy}$ beam ${ }^{-1}$ level.

V07 and to the VLA 1.4 GHz images in Feretti et al. (2005). In light of all this, the flux density values reported in Table 4 should be considered as lower limits, and any spectral consideration is deferred to a detailed ongoing multifrequency study (Giacintucci et al., in prep.).

\subsection{Clusters with complex diffuse emission}

\subsubsection{A 1682: a very complex cluster}

A $1682\left(z=0.226, \mathrm{~L}_{\mathrm{X},[0.1-2.4 \mathrm{KeV}]}=7.0 \times 10^{44} \mathrm{erg} \mathrm{s}^{-1}\right)$ is a merging massive cluster (Morrison et al. 2003) with very complex radio emission. High sensitivity and high resolution observations in the radio band (GMRT at $610 \mathrm{MHz}$ ) were first published in V08, and they show that the central compact emission visible on the NVSS is actually a blend of a number of features: (a) a strong radio galaxy, associated with the dominant $m_{\mathrm{g}}=18.0$ galaxy at the cluster centre $(z=0.21839$, Koester et al. 2007), which diffuses into a tail; (b) two filamentary structures of unclear origin, which were named $S$ - $E$ and $N$-W ridges in V08; (c) diffuse excess emission over a $\sim \mathrm{Mpc}$-size region, suggestive of the presence of an underlying giant radio halo.

Our $240 \mathrm{MHz}$ images are reported in Fig. 6. The left-hand panel shows the full resolution contours overlaid on the optical emission (red plate of the Digitized Sky Survey DSS-2), while in the right-hand panel a tapered image is overlaid on the $74 \mathrm{MHz}$ emission visible on the VLA Low-Frequency Sky Survey (VLSS). The N-W ridge extends $\sim 2^{\prime}$, i.e. $\sim 430 \mathrm{kpc}$, while E-tail is $\sim 380 \mathrm{kpc}$. The $S$-E ridge is $\sim 1^{\prime}$, i.e. $\sim 220 \mathrm{kpc}$. The brightness distribution of both the N-W and S-E ridge is centrally peaked over their full extent. 

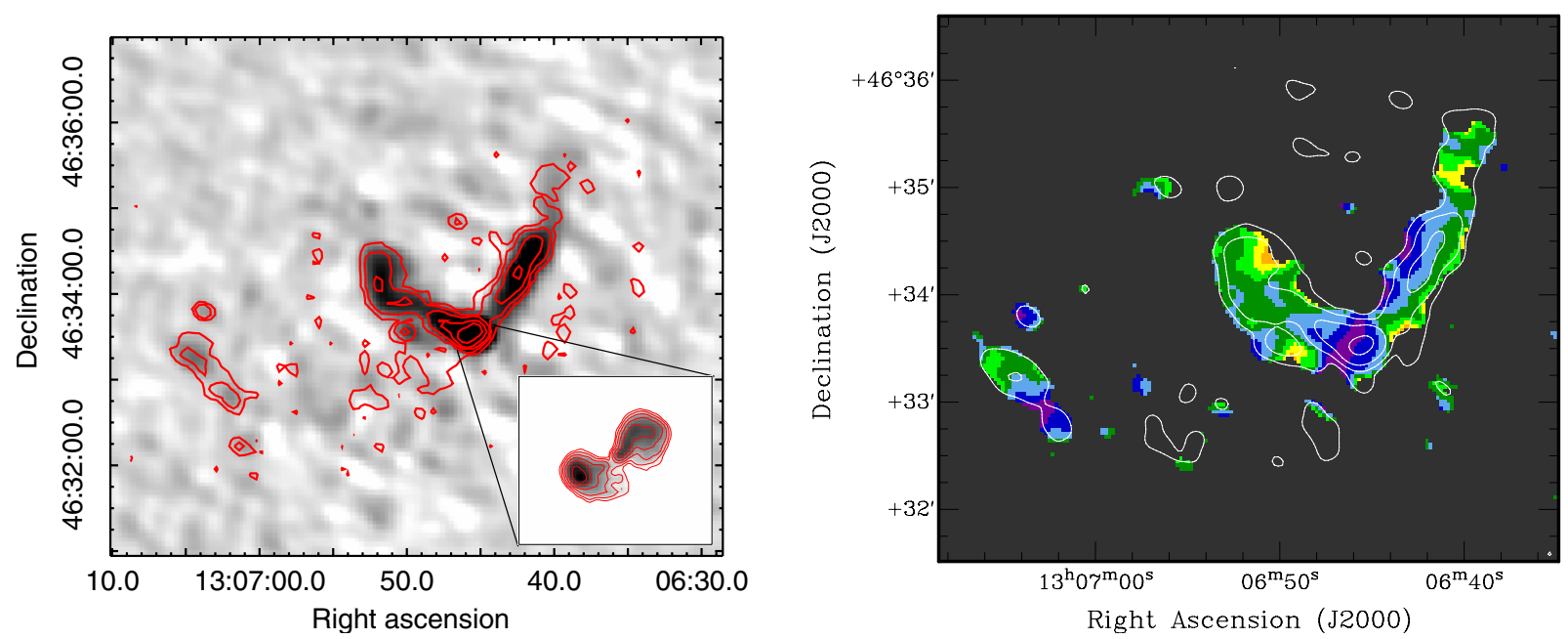

Fig. 7. Left: GMRT $240 \mathrm{MHz}$ grey-scale image of A 1682 with $610 \mathrm{MHz}$ contours overlaid. The $240 \mathrm{MHz}$ image is the same as Fig. 6, right-hand panel. The resolution of the $610 \mathrm{MHz}$ image is $6.2^{\prime \prime} \times 4.1^{\prime \prime}$, p.a. $61.2^{\circ}$, the $1 \sigma \mathrm{rms}$ in the image is $25 \mu \mathrm{Jy} \mathrm{beam}^{-1}$, the first contour is $0.1 \mathrm{mJy}^{\mathrm{m}}$ beam ${ }^{-1}$, and contours are spaced by a factor of 4 . The inset in the bottom right corner is the $1.4 \mathrm{GHz}$ VLA-A image of the central BGC at the resolution of $1.1^{\prime \prime} \times 1.1^{\prime \prime}$. Contours are spaced by a factor of 2 starting from 0.15 mJy beam ${ }^{-1}$. Right: spectral index image of A 1682, with GMRT $240 \mathrm{MHz}$ contours overlaid (resolution $12^{\prime \prime} \times 10^{\prime \prime}$, p.a. $60^{\circ}$ ) The first contour is $3 \mathrm{mJy}_{\text {beam }}^{-1}$, contours are spaced by a factor of 4 . The spectral index scale ranges from light green $\left(\alpha_{240 \mathrm{MHz}}^{610 \mathrm{MHZ}} \sim 2.3\right)$ to violet $\left(\alpha_{240 \mathrm{MHz}}^{610 \mathrm{MHZ}} \sim 0.5\right)$.

Figure 6 clearly shows the presence of a new component beyond the features already visible at $610 \mathrm{MHz}$, labelled as "diffuse component" (right-hand panel), and coincident with a similar feature on the VLSS. This component is also a prominent feature in GMRT $150 \mathrm{MHz}$ images (Macario et al. 2013) and in LOFAR (LOw Frequency ARray) observations (Macario et al., in prep.).

From the low resolution image in the right-hand panel of Fig. 6 we measured the $240 \mathrm{MHz}$ flux density of the various components and obtained $S_{(\mathrm{N}-\mathrm{W} \text { ridge })}=468 \pm 23 \mathrm{mJy}$; $S_{\text {(E-tail) }}=1126 \pm 56 \mathrm{mJy}$ (this value includes both the brightest part of the emission and the long tail, while the contribution of $\mathrm{S} 2$, associated with a 18.0 magnitude cluster galaxy at $z=0.233$, Hao et al. 2010, has been removed); $S_{(\text {S-E ridge })}=63 \pm 4 \mathrm{mJy}$; $S_{\text {(Diff Comp) }}=46 \pm 4 \mathrm{mJy}$.

The left-hand panel of Fig. 7 shows an overlay of the radio emission of A 1682 at $610 \mathrm{MHz}$ (V08) and $240 \mathrm{MHz}$. The shape, size and brightness distribution of the E-tail, the N-W, and the $\mathrm{S}-\mathrm{E}$ ridges are in very good agreement at the two frequencies.

To throw some light on the nature of the various components of the diffuse radio emission in this cluster, we performed a spectral study between $610 \mathrm{MHz}$ and $240 \mathrm{MHz}$. The total spectral index of the N-W ridge, E-tail and S-E ridge between $610 \mathrm{MHz}$ and $240 \mathrm{MHz}$, derived using images with resolution $\sim 14 "$ and computed integrating over the same area, are respectively: $\alpha_{240 \mathrm{MHz}}^{610 \mathrm{MH}}(\mathrm{N}-\mathrm{W}$ rigde $)=1.62 \pm 0.09, \alpha_{240 \mathrm{MHz}}^{610 \mathrm{MHz}}($ E-tail $)=$ $0.99_{-0.10}^{+0.09}$, and $\alpha_{240 \mathrm{MHz}}^{610 \mathrm{MHz}}(\mathrm{S}-\mathrm{E}$ rigde $)=1.53_{-0.13}^{+0.11}$. The spectrum of E-tail actually consists of two distinct components: the brightest part of the emission (before the radio emission bends) has a "normal" spectrum, i.e. $\alpha=0.78$, typical of radio galaxies, while the spectrum in the long tail is very steep, with $\alpha=2.1$.

We point out that the $610 \mathrm{MHz}$ observations we are using for this analysis do not belong to the same set of observations of the GMRT Radio Halo survey, but they are part of a longer exposure carried out two years after the survey observations. While it is still possible that the $610 \mathrm{MHz}$ flux density measurements are underestimated (see Macario et al. 2010), a check on the $\mathrm{S}-\mathrm{E}$ ridge does not suggest any missing flux at $610 \mathrm{MHz}$. The spectral index for this feature between $240 \mathrm{MHz}$ and $610 \mathrm{MHz}$ is consistent with that reported in V08 between $610 \mathrm{MHz}$ and $1.4 \mathrm{GHz}$ (NVSS).

To check for steepness gradients in the N-W ridge and in the E-tail we matched the $u-v$ coverage of the $610 \mathrm{MHz}$ and $240 \mathrm{MHz}$ datasets and produced a spectral index image of the central cluster region at the resolution of $12^{\prime \prime} \times 10^{\prime \prime}$. Our results are given in the right-hand panel of Fig. 7. The distribution of $\alpha_{240 \mathrm{MHz}}^{610 \mathrm{MHz}}$ in $\mathrm{N}-\mathrm{W}$ ridge shows a gradient parallel to the source major axis, from $\sim 1$ (dark blue) to $\sim 2.3$ (light green) going from east to west. In the E-tail two distinct regions are clearly visible: (1) the spectral index shows a gradient at the south-western end of the structure, with $\alpha_{240 \mathrm{MHz}}^{610 \mathrm{MHz}}$ steepening from $\sim 0.5$ at the location of the dominant double radio galaxy, to $\sim 1.2$ where the feature bends; (2) after the bending the spectral index is in the range $1.8-2.2$ with a patchy distribution.

To investigate the possible connection between the double radio source associated with the dominant cluster galaxy and the E-tail, we re-analysed two short archival VLA observations at $1.4 \mathrm{GHz}$ with the array in the A and D configurations (projects AM699 and AM469 respectively). The VLA-A array image at the resolution of $1^{\prime \prime}$ is reported in the inset in the left-hand panel of Fig. 7. It is clear that the BCG is a compact double, whose lobes bend in the south-west direction on an angular scale of $\sim 5^{\prime \prime}$. Such bending seems unrelated to the E-tail. The spectral index distribution along the E-tail seems to support this idea: no steepening is found moving away from the nuclear emission, as would be expected for a tailed radio galaxy.

The diffuse component south-east of the cluster centre is a new intriguing feature. It has the steepest spectrum. Comparison of the $240 \mathrm{MHz}$ and $74 \mathrm{MHz}$ flux density values is difficult, since the VLSS image provides very different values depending on the algorithm used to integrate over that portion of the image. Considering the flux density measured at $610 \mathrm{MHz}$ over the same region, we obtain a spectral index $\alpha_{610 \mathrm{MHz}}^{240 \mathrm{MHz}}=2.09 \pm 0.15$. Finally, in V08 we reported on the presence of residual flux density in a cluster region of $\sim 1 \mathrm{Mpc}$ within the centre at $610 \mathrm{MHz}$. We find a similar situation also at $240 \mathrm{MHz}$. In Venturi et al. (2011b) we carried out an analysis of this residual emission after subtracting of the individual sources from the 

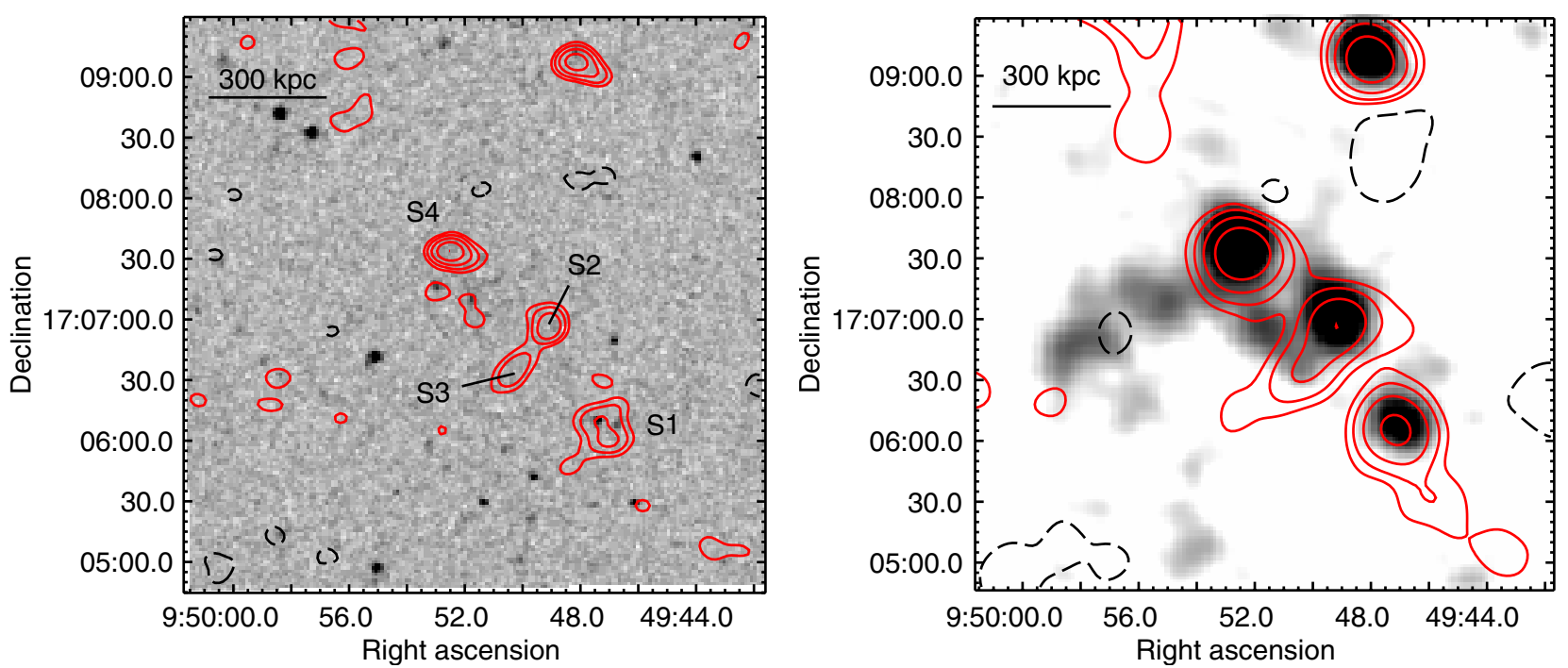

Fig. 8. Left: GMRT $325 \mathrm{MHz}$ radio contours of the emission from Z2661 at the full resolution $\left(10.8^{\prime \prime} \times 8.4^{\prime \prime}\right.$, p.a. $\left.-83^{\circ}\right)$, overlaid on the POSS-2 red image. The $1 \sigma$ noise level is $0.12 \mathrm{mJy}_{\text {beam }}{ }^{-1}$. Red contours start at $0.36 \mathrm{mJy}$ beam ${ }^{-1}$ and then scale by a factor of 2 . Black dashed contours correspond to the $-3 \sigma$ level. Individual radio galaxies are labelled from S1 to S4. Right: $325 \mathrm{MHz}$ contours at the resolution of $25.0^{\prime \prime} \times 25.0^{\prime \prime}$, p.a. $0^{\circ}$, overlaid on the GMRT $610 \mathrm{MHz}$ image $\left(17.7^{\prime \prime} \times 17.4^{\prime \prime}\right)$ from V08. Red contours start at $+3 \sigma=0.6 \mathrm{mJy}^{-1}$ beam ${ }^{-1}$ and then scale by a factor 2. The $-3 \sigma$ level is shown as black dashed contours. The rms noise level in the $610 \mathrm{MHz}$ image is $65 \mu \mathrm{Jy} \mathrm{beam}^{-1}$.

$u-v$ data, following a procedure similar to that adopted for A 781 (Venturi et al. 2011a). Excess flux density is confirmed at both frequencies and at $1.4 \mathrm{GHz}$.

To summarise, the radio emission at the centre of A 1682 is very complex, and the nature of the various features remains enigmatic. In particular,

(a) the size, morphology, and spectrum of the $\mathrm{N}-\mathrm{W}$ ridge are consistent with our knowledge of relic sources, however the spectral gradient between $610 \mathrm{MHz}$ and $240 \mathrm{MHz}$ would suggest some merger activity from west to east, which is not confirmed by the current X-ray data (see Fig. 7 in V08).

(b) On the basis of the high resolution VLA-A image of the BCG and on the spectral index distribution (Fig. 7), it seems unlikely that the E-tail is associated with the BCG, but at present there are no alternative suggestions for its origin. We note that the small-scale radio emission of the BCG hints at a motion in the southwest-northeast direction.

(c) The size of the $\mathrm{S}-\mathrm{E}$ ridge is only $\sim 220 \mathrm{kpc}$, and it is considerably smaller than the relics known so far.

(d) The nature of the diffuse component is unclear. One possibility is that it is the brightest part of an underlying steep spectrum radio halo. Alternatively, it could be a dying radio galaxy.

A detailed multifrequency study of this cluster, based on deep observations at $74 \mathrm{MHz}$ (VLA-A, Venturi et al. 2011b), at $150 \mathrm{MHz}$ (GMRT, Macario et al. 2013) and $325 \mathrm{MHz}$ (GMRT), will allow us to classify the diffuse extended sources and throw light on their nature (Dallacasa et al., in prep.).

\section{4. $Z 2661$}

Z2661 is the second most luminous cluster in the GMRT Radio Halo Cluster sample, with $L_{\mathrm{X},[0.1-2.4 \mathrm{KeV}]}=1.78 \times 10^{45} \mathrm{erg} \mathrm{s}^{-1}$. V08 reported the presence of very faint candidate diffuse emission at the cluster centre $\left(\mathrm{S}_{610 \mathrm{MHz}} \sim 5.9 \mathrm{mJy}\right)$. Our $325 \mathrm{MHz}$ observations failed to detect diffuse emission. Figure 8 shows the central cluster region at two different resolutions. Only the radio emission from the individual sources is clearly detected, and no significant excess flux density is measured over the same region covered by the diffuse emission at $610 \mathrm{MHz}$.

\subsection{The remaining clusters in the sample}

In this section, for completeness and to help the discussion along, we briefly summarise the most important radio properties of the four clusters followed up by the GMRT at $325 \mathrm{MHz}$ and $240 \mathrm{MHz}$ (Table 1), already published in separate papers.

\subsubsection{A 521: the prototype ultra-steep spectrum radio halo}

The low frequency observations of A 521, carried out at $240 \mathrm{MHz}$ and $325 \mathrm{MHz}$ to study the cluster relic (Giacintucci et al. 2008), led to the discovery of a very steep spectrum giant radio halo at the cluster centre (Brunetti et al. 2008). The source was detected in very deep $1.4 \mathrm{GHz}$ VLA observations (Dallacasa et al. 2009), which provided $\alpha_{240 \mathrm{MHz}}^{1.4 \mathrm{GHz}}=1.86 \pm 0.08$, and further imaged with the GMRT at $150 \mathrm{MHz}$ (Macario et al. 2013). The spectrum of the relic is a power law with $\alpha_{240 \mathrm{MHz}}^{4.8 \mathrm{GHz}}=1.48$ (Giacintucci et al. 2008).

\subsubsection{The ultra-steep spectrum radio halo in A697}

The GMRT $325 \mathrm{MHz}$ observations of the faint radio halo in A 697 detected at $610 \mathrm{MHz}$ (V08) revelaed that it is another example of very steep spectrum source. The $325 \mathrm{MHz}$ images and a detailed study of the radio halo and the properties of the hosting cluster were presented in Macario et al. (2010), who report a flux density value $S_{325 \mathrm{MHz}}=47.3 \pm 2.7 \mathrm{mJy}$ and a spectral index $\alpha_{325 \mathrm{MHz}}^{1.4 \mathrm{GHz}}=1.8 \pm 0.1$. This value is in line within the errors with the analysis carried out in van Weeren et al. (2011a), who reported $\alpha=1.64 \pm 0.06$ on the basis of deep $1.4 \mathrm{GHz}$ images. The steep spectrum has been confirmed by the analysis of GMRT $150 \mathrm{MHz}$ data (Macario et al. 2011b, 2013). The linear size of the source at this frequency is $\sim 1.3 \mathrm{Mpc}$, considerably larger than at higher frequencies. This giant radio halo has a smooth surface brightness to its full extent. Literature X-ray 

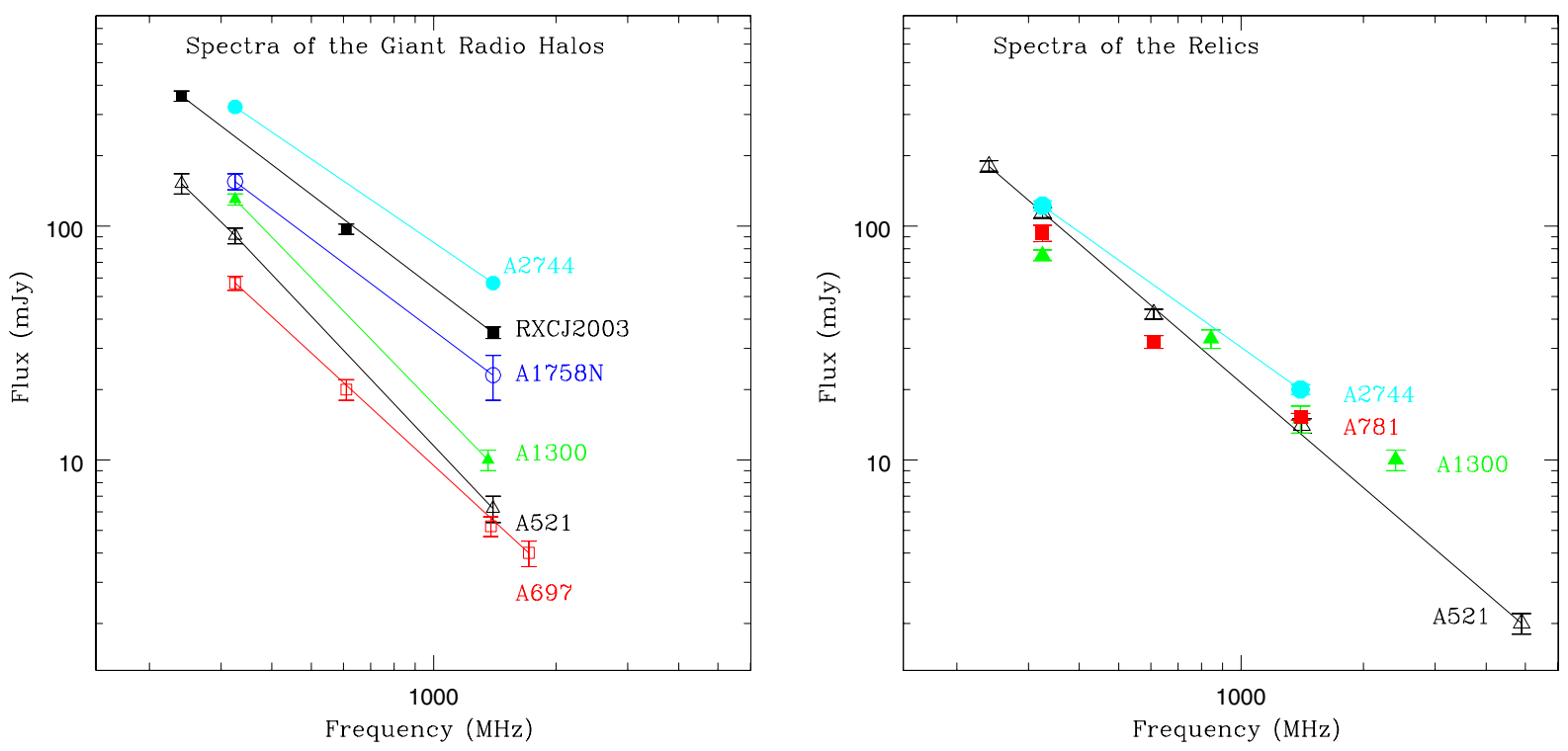

Fig. 9. Left: spectra of the giant radio haloes in the GMRT Radio Halo Survey. The $325 \mathrm{MHz}$ data points are reported in Table 4. The remaining data are taken from: A 697 Macario et al. (2010) and van Weeren et al. (2011a); A 521 Brunetti et al. (2008); RXCJ 2003.5-2323 Giacintucci et al. (2009). For A 1300 see Sect. 3.2.1; for A 1758N see Sect. 3.1.2; for A 2744 see Sect. 3.2.2. Right: spectra of relics from the GMRT radio Halo survey. The $325 \mathrm{MHz}$ data points are reported in Table 4. The remaining data are taken from: A 521 G08; A 781 Venturi et al. (2011a); A 1300 R99; A 2744 see Sect. 3.2.2. In both panels the lines are drawn to help the visual inspection and are not the best fit power law.

and optical data suggest that the cluster is unrelaxed, and even though the available information does not allow a detailed study, either a multiple merger or a major merger along the line of sight are the possible scenarios.

\subsubsection{Complex radio emission in $A 781$}

A 781 is an intriguing case. Beyond a number of individual galaxies, the central part of the cluster is dominated by a diffuse source located at the border of the X-ray emission, classified as a radio relic with spectrum $\alpha_{325 \mathrm{MHz}}^{1.4 \mathrm{GHz}}=1.25 \pm 0.06$ (V08; Venturi et al. 2011a).

On the basis of a detailed radio/X-ray analysis carried out by Cassano et al. (2010), the cluster was classified as an "outlier" in the quantitative correlations relating cluster mergers and the presence of a radio halo: the cluster shows a high degree of disturbance but lacks a radio halo. Our GMRT $325 \mathrm{MHz}$ followup observations revealed residual emission at the cluster centre, after subtracting of the individual sources (Venturi et al. 2011a). Such excess flux density sums up to $\sim 20 \mathrm{mJy}$ and is visible at the $2 \sigma$ level. Our findings did not confirm the $1.4 \mathrm{GHz}$ detection of the radio halo claimed in Govoni et al. (2011), unless the source has an unplausibly flat spectrum $\left(\alpha_{325}^{1.4 \mathrm{GHz}} \leq 0.5\right.$, see Venturi et al. 2011 a for a complete discussion).

An attractive possibility is that the residual emission reveals an underlying very low surface brightness radio halo. Allowing for all the uncertainties in our analysis, an accurate comparison between the $325 \mathrm{MHz}$ and $610 \mathrm{MHz}$ images suggests that the $325 \mathrm{MHz}$ residuals could be consistent with a diffuse source with spectrum steeper than $\alpha>1.5$.

\subsubsection{RXCJ $2003.5-2323$}

The cluster is one of the most distant in our sample $(z=$ 0.317 ) and it hosts a giant radio halo (LLS $\sim 1.4 \mathrm{Mpc}$ ), discovered with the GMRT at $610 \mathrm{MHz}$ (V07). The cluster been extensively followed by us in the radio, X-ray, and optical bands (Giacintucci et al. 2009).

The radio halo is very powerful, and its spectrum can be well fitted by a single power law with spectral index $\alpha_{240 \mathrm{MHz}}^{1.4 \mathrm{GHz}}=$ $1.27_{-0.08}^{+0.18}$. It has a regular shape, and its size is very similar from $240 \mathrm{MHz}$ to $1.4 \mathrm{GHz}$. Its brightness distribution is characterised by clumps and filaments. Our multiwavelength study supports the scenario of a merger-driven formation for this giant radio halo.

\section{Discussion}

The main goal of our GMRT $325 \mathrm{MHz}$ follow-up program of the haloes, relics, and candidates in the GMRT cluster sample (i.e. redshift range $z=0.2-0.4$, X-ray luminosity $L_{\mathrm{X}}(0.1-2.4 \mathrm{keV})>$ $5 \times 10^{44} \mathrm{erg} \mathrm{s}^{-1}, \delta>-30^{\circ}, \mathrm{V} 07$ and V08) was to perform high sensitivity imaging of diffuse cluster sources at a key frequency for our understanding of their origin. Both haloes and relics are easily detected at $325 \mathrm{MHz}$, due to their steep spectrum and increased brightness with respect to the $\mathrm{GHz}$ observing frequencies. Compared to the few other interferometers operating at frequencies of a few hundred MHz, the GMRT has the advantage to allow accurate subtraction of the individual sources usually embedded in the diffuse emission, thus allowing a much more precise flux density measurement of the diffuse cluster sources, as well as much more reliable imaging of their fine-scale features. This is critical for performing a detailed comparison with the distribution of the X-ray emission and to deriving reliable integrated spectra. As a matter of fact, integrated spectra of radio haloes still suffer from many uncertainties, mainly due to the miscellanoeus collection of data, i.e. different arrays, resolutions, and procedures in subtracting of individual embedded sources (see Venturi 2011).

Observations at frequencies $v \leq 325 \mathrm{MHz}$ clearly show that the diffuse radio emission in galaxy clusters is very complex: new features show up, beyond the canonical classification of radio haloes and relics, and the appearance of radio haloes may 
change with frequency, both in some morphological details, and in the overall size. Some of the most relevant findings in our work are summarised and discussed in the following sections.

\subsection{Morphology of radio haloes}

\subsubsection{Size versus frequency}

Our results clearly show that our view of radio haloes changes at low frequencies. In some cases, the size of radio haloes is very similar going from $1.4 \mathrm{GHz}$ down to $325 \mathrm{MHz}$ and $240 \mathrm{MHz}$. A clear example is RXCJ 2003.5-2323 (Giacintucci et al. 2009), and to a less extent A 2744. The total size of A 1300 is similar in our image and those in R99, even though the different angular resolution and sensitivity do not allow accurate comparison.

On the other hand, some radio haloes with spectral index steeper than $\alpha_{325 \mathrm{MHz}}^{1.4 \mathrm{GHz}}>1.6$ (see also next section) are more extended at frequencies of a few hundred $\mathrm{MHz}$, being barely detected at $1.4 \mathrm{GHz}$, as is the case for A 521 (Dallacasa et al. 2009).

While it is tempting to suggest that the "GHz haloes", i.e. those with "normal" $(\alpha \sim 1.2 \div 1.3)$ spectrum, tend to have sizes and shapes similar at all frequencies, and the "low frequency haloes", i.e. those with very steep spectra ( $\alpha \geq 1.6)$ show increasing size at decreasing frequency, as is the case for A 697 (see Macario et al. 2010 and 2011b, and van Weeren et al. 2011a), it is possible that this difference is the result of the limited sensitivity of the current interferometers. The improved sensitivity of the EVLA at GHz frequencies and of the LOFAR below $240 \mathrm{MHz}$ will allow us to carefully address this point, and explore the possibility that the dependence of the size with frequency is the result of the radial spectral steepening.

\subsubsection{The brightness distribution and radio/X comparison}

An important outcome of our $325 \mathrm{MHz}$ survey concerns the brightness distribution of radio haloes, which differs from case to case: A 697 is centrally peaked; A 2744 is asymmetrically peaked; most haloes have a rather "clumpy" brightness distribution (A 521, Brunetti et al. 2008; A 1300; A 1758N; RXCJ 2003.5-2323, Giacintucci et al. 2009). Other known clusters in the literature show similar properties: the Coma cluster is centrally peaked (see for instance the $325 \mathrm{MHz}$ image reported in Brown \& Rudnick 2011), while A 754 is clumpy (Macario et al. 2011a).

The comparison of the radio and X-ray brightness distribution provides further insightful information. "Clumpy" radio haloes are usually found in clusters with inhomogneous X-ray distribution, as is the case for A 521, A 1300 (see right-hand panel of Fig. 3) and RXCJ 2003.5-2323. On the other hand, the peak in the radio halo brightness distribution may coincide with the thermal peak, as is the case for A 697 (Macario et al. 2010), or be misplaced from it, as is clear in A $1758 \mathrm{~N}$ and A 2744 (right-hand panel of Figs. 2 and 4, respectively).

These observational differences may contain important information about the type of merger and on the interplay between the cluster dynamical activity and the non-thermal emission. This result should be taken into account when addressing the origin of radio haloes.

\subsection{Integrated spectra of haloes and relics}

One of the most outstanding results of our low frequency followup of the GMRT radio Halo survey has been the discovery of ultra-steep spectrum radio haloes, i.e. A521 and A 697 (see
Sects. 3.5.1 and 3.5.2, respectively). To highlight the different spectral slope of the spectrum of these two clusters compared to the others surveyed with the GMRT at $325 \mathrm{MHz}$, in the left-hand panel of Fig. 9 we report the spectra of the giant radio haloes in A 2744, A 521, A 697, A 1300, A 1758N, RXCJ 2003.5-2323. It is worth pointing out that A 521, A 697, and RXCJ 2003.5-2323 have been analysed using the same approach on the datasets at all frequencies. This is critical if we consider all the uncertainties in subtracting of the contribution of individual sources embedded in the diffuse radio emission. The lines are not the best fit, and are drawn only to help the visual inspection.

Even considering only the clusters in the GMRT sample, it is clear that the observed spectral index of radio haloes is quite different from case to case, and it goes from $\alpha_{325 \mathrm{MHz}}^{1.4 \mathrm{GHz}} \sim 1.2$ (A 2744) to $\sim 1.9$ (A 521), with a spread of at least $\Delta \alpha \sim 0.7$. This reflects on the spread in the energy distribution of the relativistic particle population of the order $\Delta \delta^{\mathrm{e}^{ \pm}} \sim 1.4$, which needs to be explained. One possibility is that a variety of mechanisms produces relativistic particles with different energy distributions. More likely, the broad range of observed synchrotron spectra suggests that the spectrum of the relativistic particles is not a power law and that it is the result of the combined effect of a high energy break in the electron energy distribution and an inhomogeneous magnetic field over the cluster volume. A break in the spectrum of the emitting particles is predicted by turbulent reacceleration models, or more generally, when the time scale of particle re-acceleration is comparable to that of radiative losses $\left(10^{7}-10^{8}\right.$ years $)$.

The observational properties of the relics in the GMRT radio halo survey (A 521, A 781, A 1300, A 2744) are more homogeneous. In all cases their morphology, brightness distribution, and extent are fairly consistent at all frequencies. The only exception is A 781, which is more extended at $325 \mathrm{MHz}$, compared to $610 \mathrm{MHz}$ and $1.4 \mathrm{GHz}$ (Venturi et al. 2011a). The spectral index $\alpha_{325 \mathrm{MHz}}^{1.4 \mathrm{GHz}}$ in the range 1.25 (A 781)-1.48 (A 521).

We do not include the double relic cluster RXCJ 1314.42515 in these spectral considerations, owing to the poor quality of the $325 \mathrm{MHz}$ images. This cluster has been re-observed with the GMRT at $325 \mathrm{MHz}$, and a separate paper is in progress (Giacintucci et al., in prep.). The spectral index between $610 \mathrm{MHz}$ and $1.4 \mathrm{GHz}$ is $\alpha_{610 \mathrm{MHz}}^{1.4 \mathrm{GHz}}=1.40 \pm 0.09$ and $1.41 \pm 0.09$ for the western and eastern relics, respectively (V07).

In the right-hand panel of Fig. 9 we report the spectra of the relics in A 2744, A 521, A 781, and A 1300. The spread in flux density for these sources is much narrower compared to haloes. The lines are drawn to help visual inspection. Due to the small number of available relic radio spectra we cannot conclude that this is a general behaviour.

\subsection{Diffuse cluster emission beyond haloes and relics}

A number of diffuse sources, which fit neither into the radio halo nor relic class, have been detected in some clusters. Our findings are summarised here below.

- A clear "bridge" of emission connects the radio halo and the relic in A 2744 (right-hand panel of Fig. 4) and in RXCJ 1314.4-2515 (V07); a similar feature is visible in A 521 (Brunetti et al. 2008) and A 1300 (right-hand panel of Fig. 3) in the region between the halo and the relic. So far radio bridges have been observed only in very few famous cases, such as the Coma cluster (Kim et al. 1989; Brown \& Rudnick 2011) and A 3667 (Carretti et al. 2013). Projection effects cannot be ruled out, however an attractive explanation 

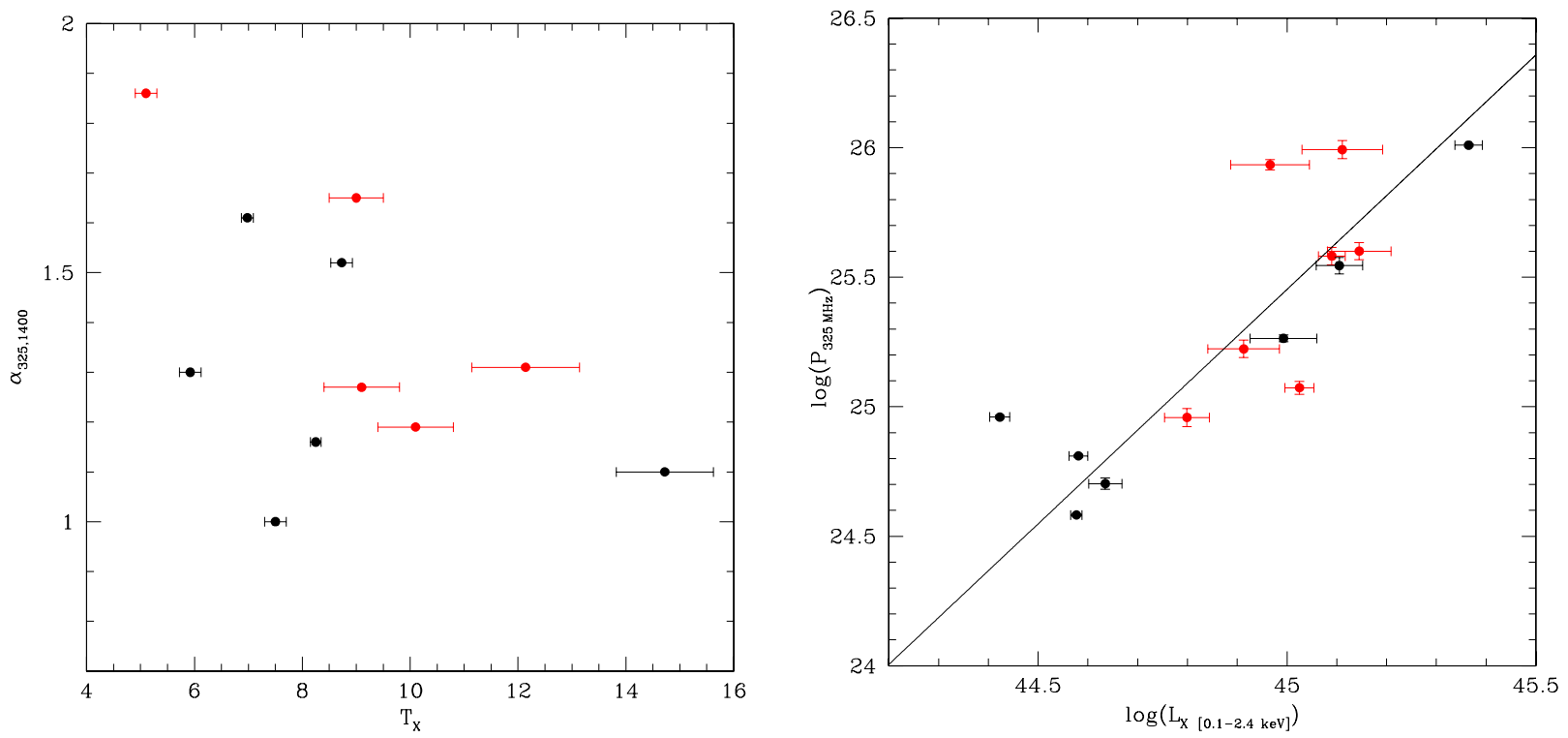

Fig. 10. Left: spectral index of the radio haloes presented in this paper and collected from the literature in the range $325 \mathrm{MHz}-1.4 \mathrm{GHz}$ plotted as function of the cluster X-ray temperature. Right: $\log L_{\mathrm{X}}-\log P_{325} \mathrm{MHZ}$ correlation. The red points in each plot are the clusters from the GMRT $325 \mathrm{MHz}$ follow up.

for these features assumes that they may come from merger shock re-accelerated electrons, which move downstream as the shock advances through the ICM and are further accelerated by the turbulence injected by the merger in the ICM closer to the cluster centre (Markevitch 2010).

- Extended emission, which cannot be easily classified, was detected in A 1300 (the "candidate relic", see Sect. 3.2.1). This source is arc-shaped and has a projected linear size of the order of $700 \mathrm{kpc}$ and radio power $\log P_{325 \mathrm{MHz}}=6.9 \times$ $10^{24} \mathrm{~W} \mathrm{~Hz}^{-1}$ (assuming that it is at the cluster distance), and it is located at the periphery of the X-ray emission.

- A 1682 is the most enigmatic cluster in our study. Three filamentary features with no obvious optical counterpart are found: the N-W ridge, the E-tail and the S-E ridge. The last is located at the boundary of the X-ray emission, but it is much smaller (at least in projection) than the typical size reported for relics, i.e. only $\sim 220 \mathrm{kpc}$, and has a radio power $\log P_{240 \mathrm{MHz}}=9.3 \times 10^{24} \mathrm{~W} \mathrm{~Hz}^{-1}$ if located at the distance of A 1682. The morphology, size, and radio power of the $\mathrm{N}-\mathrm{W}$ ridge argue in favour of a possible relic, but the direction of the steepening in the spectral index distribution is a puzzle. Our analysis of the E-tail disfavours the idea that it is related to the central double radio galaxy, even though at present there are no alternative explanations for its nature. Finally, diffuse very low surface brightness emission is visible south of the cluster centre, with total radio power $\log P_{240 \mathrm{MHz}}=6.8 \times 10^{24} \mathrm{~W} \mathrm{~Hz}^{-1}$ (assuming it is located at the distance of the hosting cluster). This radio emission could be the brightest part of an underlying more extended very low surface brightness radio halo.

- Our data suggest the presence of diffuse emission in A 781 just below the sensitivity threshold of the observations (Venturi et al. 2011a, see Table 5). This cluster deserves further investigation.

All the above results reinforce the overall finding that the diffuse cluster radio emission is more complex than the usual "radio halo" and "relic" classification when observed at frequencies $\leq 325 \mathrm{MHz}$.

\subsection{Statistical correlations for radio haloes at low frequency}

The low frequency follow up of the GMRT Radio Halo Cluster Survey has doubled the number of galaxy clusters with high sensitivity imaging of the radio halo at $325 \mathrm{MHz}$, bringing them to 14 . We are aware that the statistics is still limited, but the numbers are significant enough to start investigating some correlations.

\subsubsection{Spectral index of radio haloes and cluster temperature}

Feretti et al. (2004), and more recently Giovannini et al. (2009), claimed a possible correlation between the cluster temperature and the radio halo spectral index, suggesting that hotter clusters host radio haloes with flatter spectrum. If confirmed, this trend would provide important constraints on the origin of the emitting particles.

To investigate this point, we added all the remaining clusters in the literature to our new results with spectral information between $325 \mathrm{MHz}$ and $1.4 \mathrm{GHz}$ (A 2163, Coma, A 2255, A 665, A 2256 and A 754). Our analysis does not include A 209 and A 1300 (see Sect. 3.1.1 and 3.2.1 respectively). We used the updated values of $\alpha_{325 \mathrm{MHz}}^{1.4 \mathrm{GHz}}=1.65$ for A 697 (van Weeren et al. 2011a) and $\alpha_{325 \mathrm{MHz}}^{1.4 \mathrm{GHz}}=1.3$ for A 2255 (Pizzo \& de Bruyn 2009). These two values are considerably different than those used in the analysis of Giovannini et al. (2009), i.e. 1.2 and 1.7 for A 679 and A 2255, respectively.

In the left-hand panel of Fig. 10 we report the distribution of the clusters in the $\alpha_{325,1400}-T$ plane. We do not find any clear correlation between these two quantities. Nevertheless, at a preliminary level our plot indicates that

(1) clusters with $T<10 \mathrm{keV}$ host haloes both with flat $(\alpha \sim 1)$ and steep $(\alpha \sim 1.8)$ spectrum;

(2) clusters with $T>10 \mathrm{keV}$ host haloes with spectra flatter than $\alpha \sim 1.3$.

This behaviour is qualitatively consistent with the expectations of the re-acceleration models, which predict that radio haloes have different spectra depending on the energy released into particle re-acceleration during mergers. In this case, from simple 
enery arguments, the fraction of haloes with "flatter" spectra increases with the mass of the host cluster. In particular, if we restrict to the mass (temperature) range of our sample, moderately massive clusters $(T \sim 5-8 \mathrm{keV})$ would statistically generate both "steep" and "flat" haloes, while massive $(T>8 \mathrm{keV})$ clusters would tend to generate flatter haloes (see Cassano et al. 2006 and Cassano et al. 2010). We are aware that information on more clusters is needed to test any possible correlation between these two quantities.

\subsection{2. $\log L_{x}-\log P_{325 \mathrm{MHz}}$ correlation}

Beyond the GMRT clusters presented and discussed in this paper, we collected all the remaining clusters in the literature with high sensitivity imaging at $325 \mathrm{MHz}$ ( A2163, Coma, A 2255, A 665, A 2256, A 754, A 2219) and derived the $\log L_{\mathrm{x}}-\log P_{325 \mathrm{MHz}}$ correlation, which is shown in the right-hand panel of Fig. 10. This analysis was presented in Kempner \& Sarazin (2001) using WENSS data, and in Rudnick $\&$ Lemmerman (2009). For the first time we have derived this correlation based on deep pointed observations with accurate subtraction of foreground and background sources projected onto the radio halo emission. The best fit value of the slope is $1.81 \pm 0.28$, consistent within the errors with what is found at $1.4 \mathrm{GHz}$, i.e. $2.06 \pm 0.20$ (Brunetti et al. 2009).

Given the small size of the sample available at $325 \mathrm{MHz}$, more data are needed to confirm this correlation and to constrain its slope. Cassano (2010) shows that in the framework of the turbulent re-acceleration scenario the correlation becomes steeper and broader at the LOFAR frequencies and sensitivities.

\section{Conclusions}

The GMRT Radio Halo Cluster Survey has been the first step towards investigating of the statistical properties of radio haloes in galaxy clusters. The most relevant results can be summarised as follows. (a) The bimodal distribution of galaxy clusters with respect to the presence of a radio halo (Brunetti et al. 2007) clearly shows that radio haloes are not ubiquitous in clusters. The distribution of galaxy clusters in the $\log L_{\mathrm{X}}-\log P_{1.4 \mathrm{GHz}}$ plot provides an important piece of information for the theoretical models, suggesting that radio haloes are transient radio sources (Brunetti et al. 2009; Enßlin et al 2011) ${ }^{3}$. (b) First quantitative correlations have been found between the dynamical state of clusters and the presence of haloes, or lack thereof (Cassano et al. 2010). This result confirms that mergers play the most important role in the formation of radio haloes. (c) A relevant byproduct of the survey was the discovery of ultra-steep spectrum radio haloes. This poses new compelling constraints on the origin of these sources.

The results of the low-frequency follow up of radio haloes and relics in the GMRT radio halo cluster sample reported in this paper, not only reinforce the radio halo/cluster merger connection, but also pose new important questions.

The study of the integrated synchrotron spectrum of the radio haloes in our sample clearly shows a wide range of values for the spectral index, with $\Delta \alpha_{325 \mathrm{MHz}}^{1.4 \mathrm{GHz}} \sim 1.2 \div 1.9$, i.e. a difference of $\sim 1.4$ in the power of the distribution of the radiating particles.

\footnotetext{
3 Using an SZ-selected subsample of the GMRT clusters, obtained from the first Planck cluster catalogue, Basu (2012) found that the cluster radio bimodality becomes considerably weaker in the $Y_{\mathrm{ZS}}-\log P$ plot, raising additional questions on the evolution of radio haloes.
}

This result, together with our points (a) and (b) above, is consistent with the expectations of the turbulent re-acceleration model, which makes the unique prediction of a high-frequency break in the spectrum of the electrons emitting at radio frequencies. This implies a range of spectral slopes in the spectra of radio haloes, including the ultra steep ones. At a more general level, these observational results suggest that inefficient mechanisms of particle acceleration activated during cluster mergers play an important role in the generation of radio haloes.

We found differences in the surface brightness distribution of the radio haloes. Some of them are clumpy, others are centrally peaked, and in other cases the isodensity contours are asymmetrically peaked. In some clusters we found a misplacement between the radio and X-ray peaks of emission, which deserves further investigation.

Bridges of emission, connecting (at least in projection) the relic and the radio halo emission were found in A 521, A 1300, A 2744, and RXCJ 1314.5-2515. Such features can be explained in the context of merger shocks and turbulence during merger processes (Markevitch 2010).

Our $325 \mathrm{MHz}$ survey has doubled the number of clusters with high sensitivity imaging of the diffuse cluster scale emission at this frequency. This allowed us to start investigating some statistical properties of radio haloes at low frequencies. For the first time we derived the correlation between the radio power at $325 \mathrm{MHz}$ and the X-ray cluster luminosity by means of deep pointed observations. The slope is consistent within the errors with that derived at $1.4 \mathrm{GHz}$, however the correlation needs to be constrained on a more solid basis with more data.

Finally, we investigated a possible trend between the radio halo spectral index and the temperature of the hosting cluster using the new data presented in the paper and updated information from the literature. With the data available at present, we cannot confirm there is the $\alpha-T$ correlation claimed in Giovannini et al. (2009).

Our study shows that systematic high sensitivity, low frequency imaging of diffuse cluster radio sources is the next step to take to further improve our knowledge of the mechanisms at the origin of their formation.

Acknowledgements. We thank the staff of the GMRT for their help during the observations. GMRT is run by the National Centre for Radio Astrophysics of the Tata Institute of Fundamental Research. We acknowledge financial contribution from the Italian Ministry of University and Research, from MIUR grants PRIN2005 and 2006, from PRIN-INAF2005 and PRIN-INAF2008, and from contract ASI-INAF I/023/05/01. Support for S.G. was provided by NASA through Einstein Postdoctoral Fellowship Award Number PF0-110071 issued by the Chandra X-ray Observatory Center, which is operated by the Smithsonian Astrophysical Observatory. G.M. acknowledges financial support by the Agence Nationale de la Recherche through grant ANR-09-JCJC-0001-01.

\section{References}

Akamatsu, H., Takizawa, M., Nakazawa, K., et al., 2012, APSJ, 64, 67 Bacchi, M., Feretti, L., Giovannini, G., et al. 2003, A\&A, 400, 465 Basu, K. 2012, MNRAS, 421, L112

Blasi, P., \& Colafrancesco, S. 1999, A. Ph., 12, 1999

Böhringer, H., Schuecker, P., Guzzo, L., et al. 2004, A\&A, 425, 367

Bonafede, A., Feretti, L., Giovannini, G., et al. 2009, A\&A, 503, 707

Bondi, M., Ciliegi, P., Venturi, T., et al. 2007, A\&A, 463, 519

Boschin, W., Girardi, M., Barrena, R., et al. 2012, A\&A, 340A, 143

Bourdin, H., Mazzotta, P., Markevitch, M., et al. 2012, ApJ, submitted

Brown, S., \& Rudnick, L. 2011, MNRAS, 412, 2

Brunetti, G. 2011, Mem. Soc. Astron. It., 82, 515

Brunetti, G., \& Blasi, P. 2005, MNRAS, 363, 1173

Brunetti, G., \& Lazarian, 2012, MNRAS, 412, 817

Brunetti, G., Setti, G., Feretti, L., \& Giovannini, G. 2001, MNRAS, 320, 365

Brunetti, G., Venturi, T., Dallacasa, D., et al. 2007, ApJ, 670, L5

Brunetti, G., Giacintucci, S., Cassano, R., et al. 2008, Nature, 455, 944 
Brunetti, G., Cassano, R., Dolag, K., et al. 2009, A\&A, 507, 661 Buote, D. A. 2001, ApJ, 553, 15

Carretti, E., Brown, S., Stavaley-Smith, L., et al. 2013, MNRAS, in press [arXiv: 1205.1082v1]

Cassano, R. 2009, in The low frequency radio Universe, eds. D. J. Saikia,

D. A. Green, Y. Gupta, \& T. Venturi, ASP Conf. Ser., 407, 223

Cassano, R. 2010, A\&A, 517A, 10

Cassano, R., \& Brunetti, G. 2005, MNRAS, 357, 1313

Cassano, R., Brunetti, G., \& Setti, G. 2006, MNRAS, 369, 1577

Cassano, R., Brunetti, G., Venturi, T., et al. 2008, A\&A, 480, 687

Cassano, R., Ettori, S., Giacintucci, S., et al. 2010, ApJ, 721, L82

Cassano, R., Brunetti, G., \& Venturi, T. 2011, JApA, 32, 519

Clarke, T. E., \& Enßlin, T. A. 2006, AJ, 131, 2900

Dallacasa, D., Brunetti, G., Giacintucci, S., et al. 2009, ApJ, 699, 1288

David, L. P., \& Kempner, J. 2004, ApJ, 613, 840

Dennison, B. 1980, ApJ, 239, L93

Dwarakanath, K. S., Rudnick, L., Shankar, N. U., \& Venturi, T. 2011, Diffuse Relativistic Plasmas, Bangalore, 1-4 March 2010, Conf. Proc. (Indian Academy of Sciences), JApA, 32, 4

Enßlin, T., \& Gopal-Krishna, 2001, A\&A, 366, 26

Enßlin, T. A., Bierman, P. L., Klein, U., et al. 1998, A\&A, 332, 395

Enßlin, T., Pfrommer, C., Miniati, F., et al. 2011, A\&A, 527, A99

Feretti, L., Brunetti, G., Giovannini, G., et al. 2004, JKAS, 37,315

Feretti L., Schuecker, P., Böringer, H., et al. 2005, A\&A, 444, 157

Feretti, L., Giovannini, G., Govoni, L., et al. 2012, A\&ARv, 20, 54

Ferrari, C., Govoni, F., Schindler, S., et al. 2008, SSRv, 134, 93

Ferrari, C., Brüggen, M., Brunetti, G., \& Venturi, T. 2011, Mem. Soc. Astron. It., 82,3

Finoguenov, A., Sarazin, C. L., Nakazawa, K., et al. 2010, ApJ, 715, 1143

Fujita, Y., Takizawa, M., \& Sarazin, C. L. 2003, ApJ, 584, 190

Geller, M. J., Dell'Antonio, I. P., Kurtz, M. J., et al. 2005, ApJ, 635L, 125

Giacintucci, S., Venturi, T., Macario, G., et al. 2008, A\&A, 486, 347

Giacintucci, S., Venturi, T., Brunetti, G., et al. 2009, A\&A, 505, 45

Giacintucci, S., Cassano, R., Brunetti, G., et al. 2012, ApJ, submitted (G12)

Giovannini, G., Bonafede, A., Feretti, L., et al. 2009, A\&A, 507, 1257

Girardi, M., \& Mezzetti, M. 2001, ApJ, 548, 79

Govoni, F., Feretti, L., Giovannini G., et al. 2001, A\&A, 376, 803

Govoni, F., Markevitch, M., Vikhlinin A., et al. 2004, ApJ, 605, 695

Govoni, F., Murgia, M., Giovannini, G., et al. 2011, A\&A, 529, A69

Hao, J., McKay, T. A., Koester, B., et al. 2010, ApJS, 191, 254

Hoeft, M., \& Brüggen, M. 2007, MNRAS, 375, 77

Jaffe, W. J. 1977, ApJ, 216,212

Kempner, J. C., \& David, L. P. 2004, MNRAS, 385, 392

Kempner, J. C., \& Sarazin, C. L. 2001, ApJ, 548, 639

Keshet, U., \& Loeb, A. 2010, ApJ, 722, 737

Kim, K.-T., Kronberg, P. P., Giovannini, G., et al. 1989, Nature, 341, 720
Koester, B. P., McKay, T. A., Annis, J., et al. 2007, ApJ, 660, 239 Lemonon, L., Pierre, M., Hunstead, R. W., et al. 1997, A\&A, 326, 34 Liang, H., Hunstead, R. W., Birkinshaw, M., et al. 2000, ApJ, 544, 686 Macario, G., Venturi, T., Brunetti, G., et al. 2010, A\&A, 517, A43

Macario, G., Markevitch, M., Giacintucci, S., et al. 2011a, ApJ, 728, 82

Macario, G., Venturi. T., Dallacasa D., et al. 2011b, Mem. Soc. Astron. It., 82, 557

Macario, G., Venturi, T., Intema, H., et al. 2013, A\&A, in press

Markevitch, M. 2010, 12th Marcel Grossman Meeting, Paris [arXiv: 1010.3660v1]

Markevitch, M., Gonzalez, A. H., David, L., et al. 2002, ApJ, 567, 27

Markevitch, M., Govoni, F., Brunetti, G., et al. 2005, ApJ, 627, 733

Mazzotta, P., Bourdin, H., Giacintucci, S., et al. 2011, Mem. Soc. Astron. It., 82, 495

Merten, J., Coe, D., Dupke, R., et al. 2011, MNRAS, 417, 333

Morrison, G. E., Owen, F. N., Ledlow, M. J., et al. 2003, ApJSS, 146, 267

O'Dea, C. P., \& Owen, F. N. 1985, AJ, 90, 954

O’Dea, C. P., Sarazin, C. L., \& Owen, F. N. 1877, ApJ, 316, 113

Ogrean, G., Brüggen, M., Röttgering, H., et al. 2013, MNRAS, 429, 2617

Orrú, E., Murgia, M., Feretti, L., et al. 2007, A\&A, 467, 943

Owers, M. S., Couch, W. J., Nulsen, P. E. J., et al. 2012, ApJ, 750, L23

Petrosian, V. 2001, ApJ, 577, 560

Pfrommer, C., \& Enßlin, T. A. 2004, JKAS, 37, 455

Pierre, M., Oukbir, J., Dubreuil, D., et al. 1997, A\&AS, 124, 283

Pizzo, R., \& de Bruyn, A. G. 2009, A\&A, 507, 639

Ragozzine, B., Clowe, D., Markevicth, M., et al. 2012, ApJ, 744, 94

Reid, A. D., Hunstead, R. W., Lemonon, L., et al. 1999, MNRAS, 302,571 (R99)

Roland, J. 1981, A\&A, 93, 407

Rudnick, L., \& Lemmerman, J. A. 2009, ApJ, 697, 1341

Schlickeiser, R., Sievers, A., \& Thiemann, H. 1987, A\&A, 182, 21

Valtchanov, I., Murphy, T., Pierre, M., et al. 2002, A\&A, 392, 795

van Weeren, R. J., Röttgering, H. J. A., Brüggen, M., \& Hoeft, M. 2010, Science, 330,347

van Weeren, R. J., Brüggen, M., Röttgering, H. J. A., et al. 2011a, A\&A, 533, A35

van Weeren, R., Intema, H. T., Röttgering, H. J. A., et al. 2011b, Mem. Soc. Astron. It., 75, 282

van Weeren, R. J., Bonafede, A., Ebeling, H., et al. 2012, MNRAS, 425, L36

Venturi, T. 2011, Mem. Soc. Astron. It., 82, 499

Venturi, T., Bardelli, S., Zagaria, M., et al. 2002, A\&A, 385, 39

Venturi, T., Giacintucci, S., Brunetti, G., et al. 2007, A\&A, 463, 937 (V07)

Venturi, T., Giacintucci, S., Dallacasa, D., et al. 2008, A\&A, 484, 327 (V08)

Venturi, T., Giacintucci, S., Dallacasa, D., et al. 2011a, MNRAS, 414, L65

Venturi, T., Giacintucci, S., Dallacasa, D. 2011b, JApA, 32, 501

Ziparo, F., Braglia, F. G., Pierini, D., et al. 2012, MNRAS, 420, 2480 